\title{
Bacteriochlorophyll and Carotenoid Excitonic Couplings in the LH2 System of Purple Bacteria
}

\author{
Sergei Tretiak* \\ Theoretical Division, Los Alamos National Laboratory, Los Alamos, New Mexico 87545 \\ Chris Middleton, Vladimir Chernyak, and Shaul Mukamel* \\ Department of Chemistry, University of Rochester, P. O. RC Box 270216, Rochester, New York 14627-0216
}

Received: April 25, 2000; In Final Form: June 26, 2000

\begin{abstract}
An effective Frenkel-exciton Hamiltonian for the entire LH2 photosynthetic complex (B800, B850, and carotenoids) from Rhodospirillum molischianum is calculated by combining the crystal structure with the Collective Electronic Oscillators (CEO) algorithm for optical response. Electronic couplings among all pigments are computed for the isolated complex and in a dielectric medium, whereby the protein environment contributions are incorporated using the Self-Consistent Reaction Field approach. The absorption spectra are analyzed by computing the electronic structure of the bacteriochlorophylls and carotenoids forming the complex. Interchromophore electronic couplings are then calculated using both a spectroscopic approach, which derives couplings from Davydov's splittings in the dimer spectra, and an electrostatic approach, which directly computes the Coulomb integrals between transition densities of each chromophore. A comparison of the couplings obtained using these two methods allows for the separation of the electrostatic (Förster) and electron exchange (Dexter) contributions. The significant impact of solvation on intermolecular interactions reflects the need for properly incorporating the protein environment in accurate computations of electronic couplings. The Förster incoherent energy transfer rates among the weakly coupled B800-B800, B800-B850, Lyc-B850, and Lyc-B850 molecules are calculated, and the effects of the dielectric medium on the LH2 light-harvesting function are analyzed and discussed.
\end{abstract}

\section{Introduction}

The primary process of photosynthesis involves the creation of chemical free-energy by capturing sunlight. ${ }^{1-2}$ The atomic structure of the light-harvesting apparatus in purple bacteria has been recently constructed through a combination of X-ray crystallography (Rhodopseudomoas (Rps.) acidophila ${ }^{3}$ and Rhodospirillum (Rs.) molischianum), ${ }^{4}$ electron microscopy (Rhodospirillum rubrum ${ }^{5}$ and Rhodovulum sulfidophilum), ${ }^{6-7}$ and modeling. The photosynthetic unit (PSU) contains two types of light-harvesting complexes: LH1, which surrounds the reaction center $(\mathrm{RC}),{ }^{8}$ and $\mathrm{LH} 2$, which is not in direct contact with the RC but transfers energy to the reaction center through LH1, where it is subsequently converted into a stable charge separation state. ${ }^{9-15}$ This organization is common to both photosynthetic bacteria and higher plants.

LH2 of Rs. molischianum is made out of $\alpha \beta$-heterodimers. Its pigment structure is shown in Figure 1, and the chromophore labeling scheme is given in Figures 3, 4, and 5. The complex is a circular aggregate made out of eight units, each consisting of a short peptides pair ( $\alpha$ and $\beta$ apoproteins), three Bacteriochlorophylls-a (Bchls- $a$ ) molecules and one (or two) carotenoid. Bchls- $a$ molecules form two rings labeled by their absorption maxima (B850 and B800), bridged by carotenoids (see Figure 1 ). The Bchls- $a$ perform the primary light harvesting function: absorbing light and transferring the resulting electronic excitations toward the RC. ${ }^{9-12}$ This energy funnel is further supplemented with excitations absorbed by carotenoids. Slightly structurally different carotenoids are found in various PSU such as Lycopene (Lyc) in Rs. molischianum ${ }^{4}$ and Rhodopsin Glucosite in Rps. acidophila. ${ }^{3}$ In addition to light-harvesting, these molecules play diverse roles in photosynthesis such as photoprotection (quenching triplets, thus preventing the formation of harmful singlet oxygen), and structure stabilization.

Understanding the organization and functionality of the PSU is a major goal of photosynthetic research. Numerous spectroscopic optical measurements ${ }^{16-32}$ provide microscopic probes for the energy capture and transfer mechanisms of the BChls and Carotenoids. The energetic ladder and possible energy transfer channels within the LH2 complex are shown in Figure 2. Each Bchl- $a$ has several low-frequency intense absorbing states: $Q_{y}, Q_{x}$, and $B_{x}$. The carotenoid has an optically forbidden $S_{1}$ state with near $A_{g}$ symmetry, whereas the next $S_{2}$ state with $B_{u}$ symmetry is strongly allowed in linear absorption. The energy gradient (Figure 2) in LH2 leads to excitation transfer to the $Q_{y}$ band of the upper B850 ring prior to the next photosynthetic step (i.e., transferring energy to the LH1 complex). The role of Soret (B) states in photosynthesis has not been clearly established. However, femtosecond internal conversion from $B_{x}$ and $Q_{x}$ to $Q_{y}$ is possible for the individual Bchls- $a$. The excitations of $\mathrm{B} 800$ could then be transferred among the lower ring chromophores (B800-B800) in $0.8-0.9$ ps $^{10}$ in Rs. molischianum (0.3-0.5 ps ${ }^{10,21,30}$ in Rps. acidophila) and further to the upper ring $(\mathrm{B} 800-\mathrm{B} 850)$ in $0.6-0.7 \mathrm{ps}^{19-21}$ (in $R s$. sphaeroides and Rps. acidophila). Much effort has been devoted 


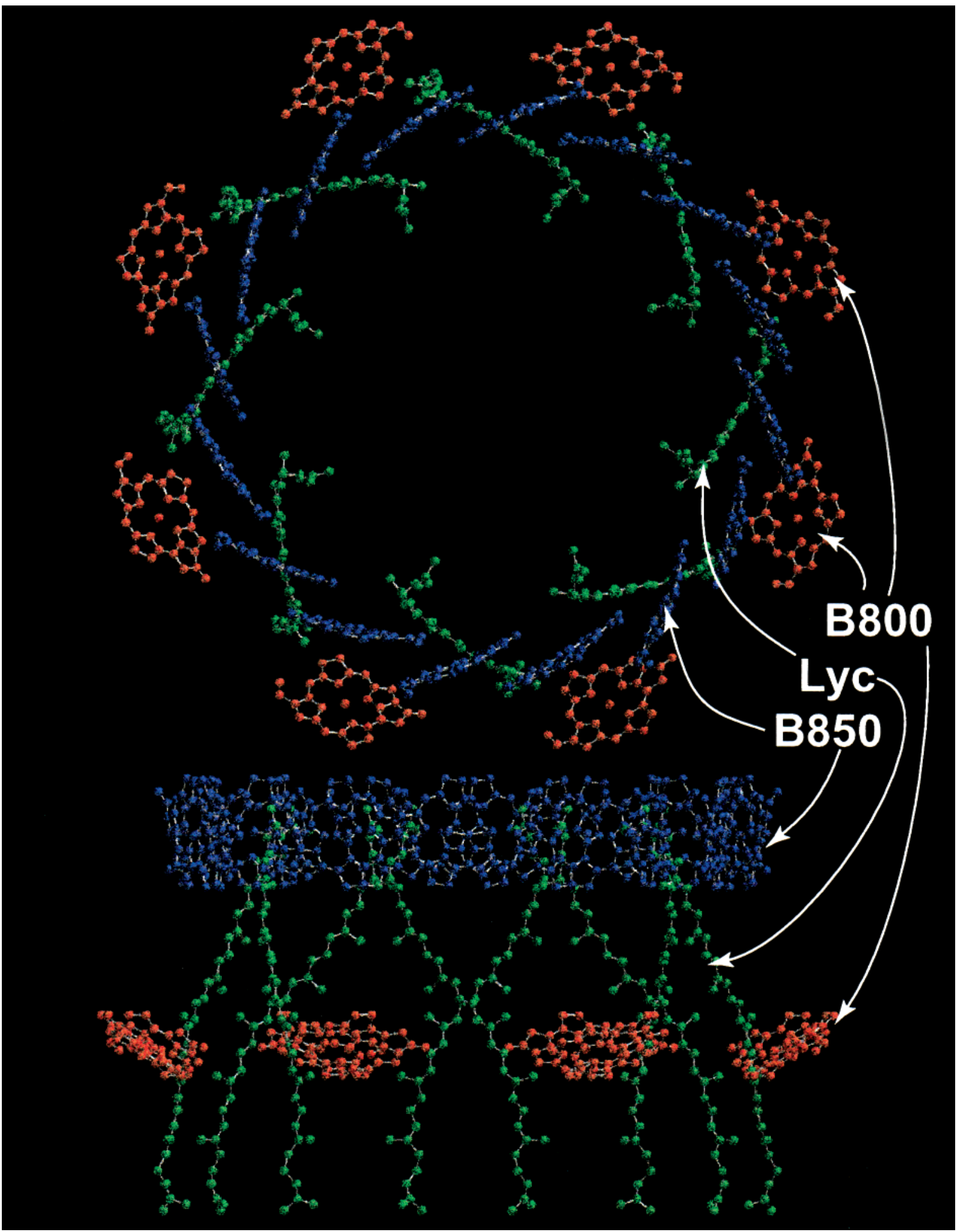

Figure 1. Top and side view of pigments in the Light Harvesting Complex II (LH2) of Rs. molischianum. The aggregate is made of the upper ring of 16 Bchls- $a$ (blue) paired in $8 \alpha \beta$ heterodimers or intrasubunits (B850 molecules), lower ring of 8 Bchls- $a$ (red) (B800 molecules), and ring of 8 lycopenes (carotenoids) (green).

to studying the energy transfer from the $1 B_{u}$ state of carotenoid. Two distinct pathways have been identified: internal conversion to the $2 A_{g}$ state followed by transfer to the $Q_{y}$ state of Bchl- $a$, or direct intermolecular excitation hopping $1 B_{u}-Q_{x}$ and $1 B_{u}-$ $Q_{y}$. Initial studies have suggested the former path. ${ }^{33,10}$ However, more recent investigations indicate that the second channel can effectively compete with the internal conversion. ${ }^{34,19}$ The total depopulation time of the $1 B_{u}$ state is founded to be $\sim 50-100$ fs. ${ }^{35,36}$ Experimental study of several PSU has also shown that $\sim 75 \%$ of the energy absorbed by the carotenoids is transferred to B850, whereas the remaining part initially goes to B800 and finally ends up on B850 through the B800-B850 relaxation channel. ${ }^{37,38}$

These energy-migration pathways in antenna complexes depend on the electronic couplings between the donor and the acceptor chromophores. For the weakly coupled B800-B800, 


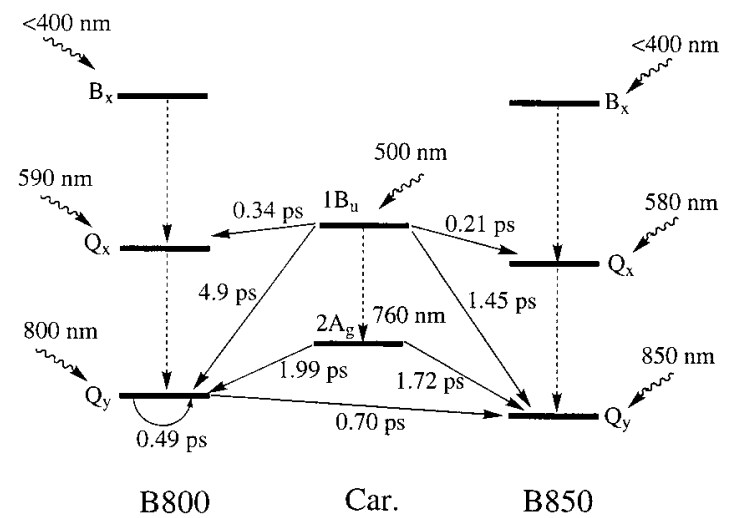

Figure 2. Electronic energy levels, major excitation funneling pathways, and their calculated transfer rates in LH2 of Rs. molischianum. Internal conversion is represented by dashed arrows, whereas interpigment energy flows are shown by solid arrows. Wavy arrows point to the light-harvesting states. Calculated Förster transfer rates along these channels are also given in Table 5 .

B800-B850, Lyc-B850, and Lyc-B850 molecules, energy transfer may be described ${ }^{34,10}$ by the Förster incoherent hopping mechanism. ${ }^{40,36}$ On the other hand, because B850-B850 couplings are strong, exceeding the energetic disorder, the molecular exciton picture should be used for the description of energy migration in the upper B850 ring. ${ }^{41-43}$ In either case, the key parameters are the electronic couplings among chromophores and considerable theoretical effort has been devoted to their calculation.

The point dipole approximation (PDA),${ }^{44-10}$ which assumes that the chromophore sizes are small compared to their separation, is routinely used for evaluating coupling constants. This gives only a crude estimate of couplings between Bchls- $a$ and completely fails to describe carotenoids in the LH2 system $^{34,39}$ because the chromophore sizes $(L \approx 9 \AA$ for Bchl- $a$ and $\mathrm{L} \approx$ $27 \AA$ for Lyc) are not small compared to their separations. Avoiding the PDA and taking into account the Coulomb interaction between the actual charge distributions poses no conceptual difficulty and has been employed in recent calculations. ${ }^{16,34,35,45,46}$ Tables 2, 3, and 4 summarize current computations of LH2 couplings. Several effective Hamiltonians have been computed for isolated Bchl- $a$ aggregates (Table 2) (medium effects are only crudely incorporated by including the macroscopic refractive index in the PDA expressions for the coupling constants $\left.{ }^{34,10}\right)$. Most calculations were performed for the Rps. acidophila complex (Table 2, columns d-k); fewer results exist for the Rs. molischianum (Table 2, columns a-c) whose crystal structure was reported more recently. The pointmonopole approach computes interactions between transition monopoles distributed on the atomic centers at the Configuration Interaction Singles (CIS) level using the simplified PPP Hamiltonian. ${ }^{16}$ In a separate study, excitonic couplings in $R s$. molischianum were obtained by fitting effective Hamiltonian parameters to electronic spectra obtained with extensive semiempirical INDO/S/CIS calculations of the upper and lower rings. ${ }^{47,48}$ The cost of such calculations grows very rapidly with system size. The method has been applied to each ring separately, and the interactions between the B800 and B850 rings which requires computation of the entire LH2 aggregate have not been reported yet. The INDO/S/CIS overestimates transition dipoles of Bchls- $a^{48}$ and consequently also the coupling parameters (Table 2 , column b). One possible reason for the discrepancy is that the dielectric medium has been mostly ignored in these calculations. The absorption spectra of Bchls- $a$ monomer and dimer were studied with semiempirical INDO/
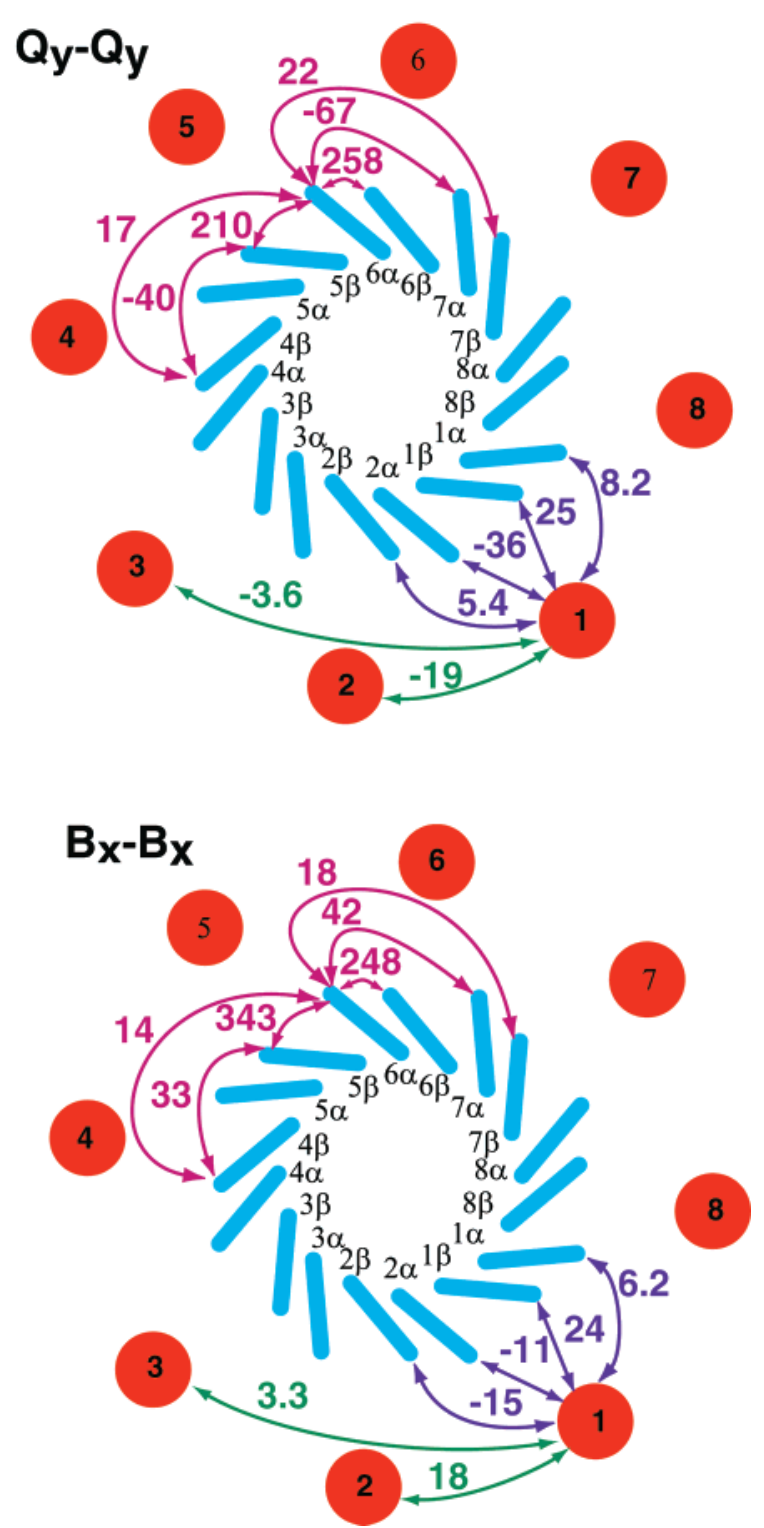

Figure 3. Graphical representation of the calculated excitonic couplings (in $\mathrm{cm}^{-1}$ ) in LH2 of Rs. molischianum shown in Figure 1 in the dielectric medium. These couplings are also given in Tables 2 and 3.

S/CIS calculations using Onsager's self-consistent reaction field SCRF approach to model the effects of protein environment. ${ }^{48}$ However, no attempt has been made to analyze the dielectric medium effects on the electronic couplings. Recent INDO/S/ CIS calculations of electronic couplings from splittings in Bchl- $a$ dimer spectra from Rps. acidophila ${ }^{46}$ explicitly included the nearest protein environment to model the local fields effects. Significant dielectric effects on the Bchl- $a$ interactions have been reported (see column $\mathrm{k}$ in Table 2).

Because of difficulties in the theoretical treatment of the entire LH2 system, only few systematic theoretical investigations of carotenoid interactions with other pigments have been reported (Table 4). Most recently, Schulten's ${ }^{39}$ and Fleming's ${ }^{35,34,39}$ groups have studied the isolated Rs. molischianum and Rps. acidophila complexes, respectively. The need for an excitonic modeling of the $\mathrm{LH} 2$ upper ring for describing the excitation transfer between carotenoids and Bchls- $a$ was argued in refs 49 and 41 . The electrostatic interaction between transition densities of each chromophore was recently calculated at the $\mathrm{ab}$ initio level with configuration interaction singles (CIS). ${ }^{34,39}$ 


\section{$1 B_{u}-Q_{y}$}
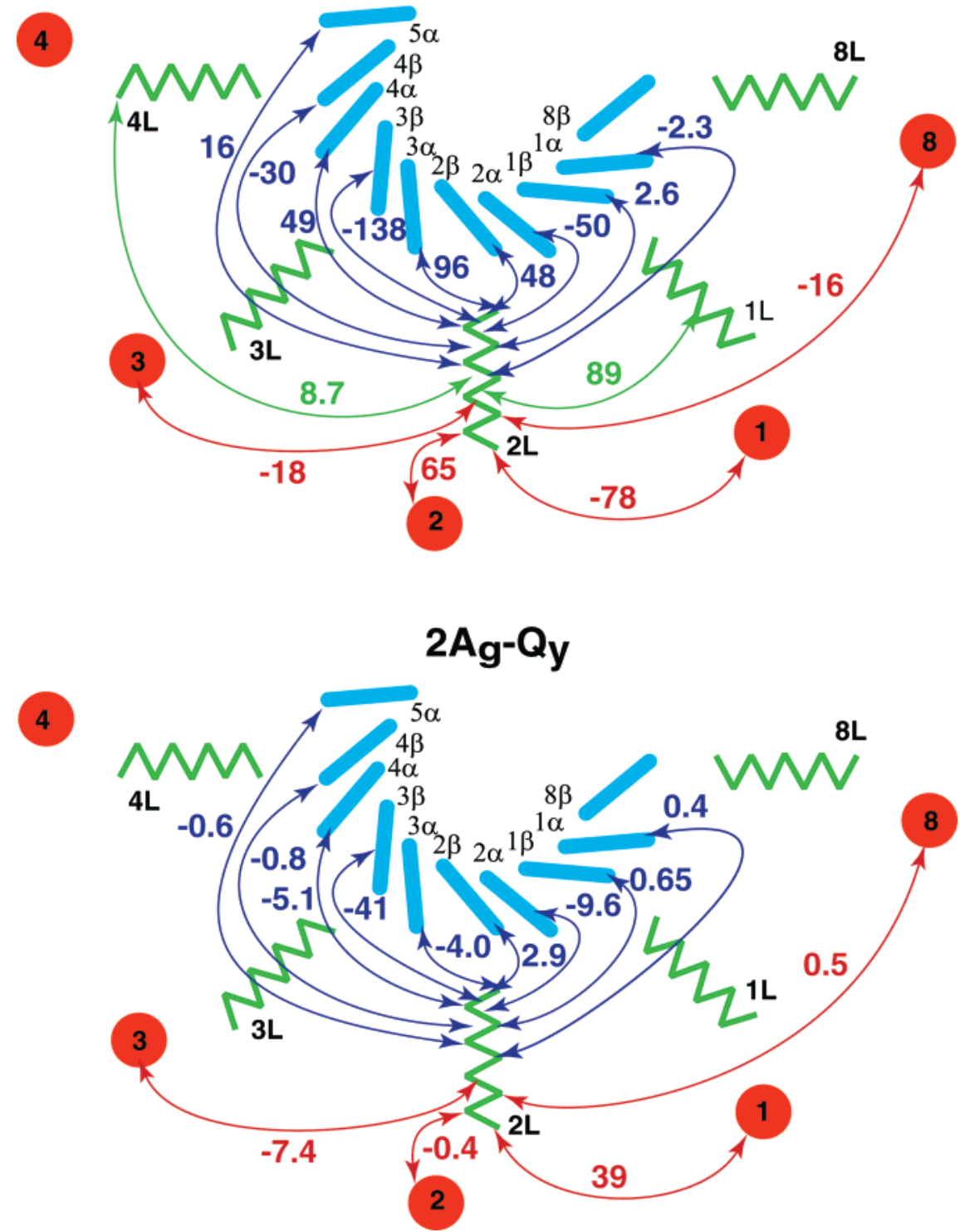

Figure 4. Graphical representation of the calculated excitonic couplings (in $\mathrm{cm}^{-1}$ ) in LH2 of Rs. molischianum shown in Figure 1 in the dielectric medium. These couplings are also given in Table 4.

As expected, this study found significant deviation from the PDA for Rps. acidophila complex (compare columns e and $\mathrm{f}$ in Table 2, and columns c and d (e and f) in Table 4). However, these expensive ab initio/CIS calculations still required rescaling the magnitudes of the chromophores' transition dipole moments, (e.g., by a factor of 1.5 for $Q_{y}$ ) in order to match experiment. The combined effect of the protein environment and carotenoids has not been computed yet.

In a recent article, ${ }^{50}$ we reported the effective Hamiltonian (column a in Table 2) for isolated Bchls- $a$ aggregates in the Rs. molischianum LH2 complex calculated using the collective electronic oscillator (CEO) approach. ${ }^{51,52}$ The parameters obtained from splittings in dimer spectra give an adequate estimate for Förster energy transfer among Bchls- $a$ without further rescaling. In this article, we apply the same approach to compute interchromophore electronic interactions among Bchls- $a$ and Lyc in LH2 complex of Rs. molischianum in a dielectric medium. The CEO algorithm computes the optical response directly by solving equations of motion; excited state wave functions are never calculated explicitly. ${ }^{53-57}$ To study the effects of protein environment, we use the Onsager solvation model combined with the self-consistent reaction field treatment. Comparison of the dielectric medium and isolated aggregate results clearly demonstrates the environmental impact on the linear absorption spectra and intermolecular interactions. We employ two different techniques for calculating the electronic couplings: a spectroscopic procedure, which is based on computing the Davydov's splitting in the dimer spectrum, ${ }^{50}$ and a Coulombic method, which computes the electrostatic interactions between transition densities of individual pigments. Because the former includes both Coulomb and exchange interactions, whereas the latter only incorporates the Coulomb interactions, a comparison of the results allows the separation electrostatic and electron exchange contributions to interchromophore couplings.

The effective Frenkel exciton Hamiltonian computed here accounts for protein solvation effects and describes the interactions among all Bchl- $a$ and Lyc pigments of LH2. It includes the complete set of parameters for $Q_{y}-Q_{y}$ and $B_{x}-B_{x}$ bands of Bchl- $a$ aggregates, $1 B_{u}-Q_{y}, 1 B_{u}-Q_{x}, 1 B_{u}-B_{x}$, and $2 A_{g}-Q_{y}$ bands of Lyc-Bchl system, $1 B_{u}-1 B_{u}$ and $2 A_{g}-2 A_{g}$ 

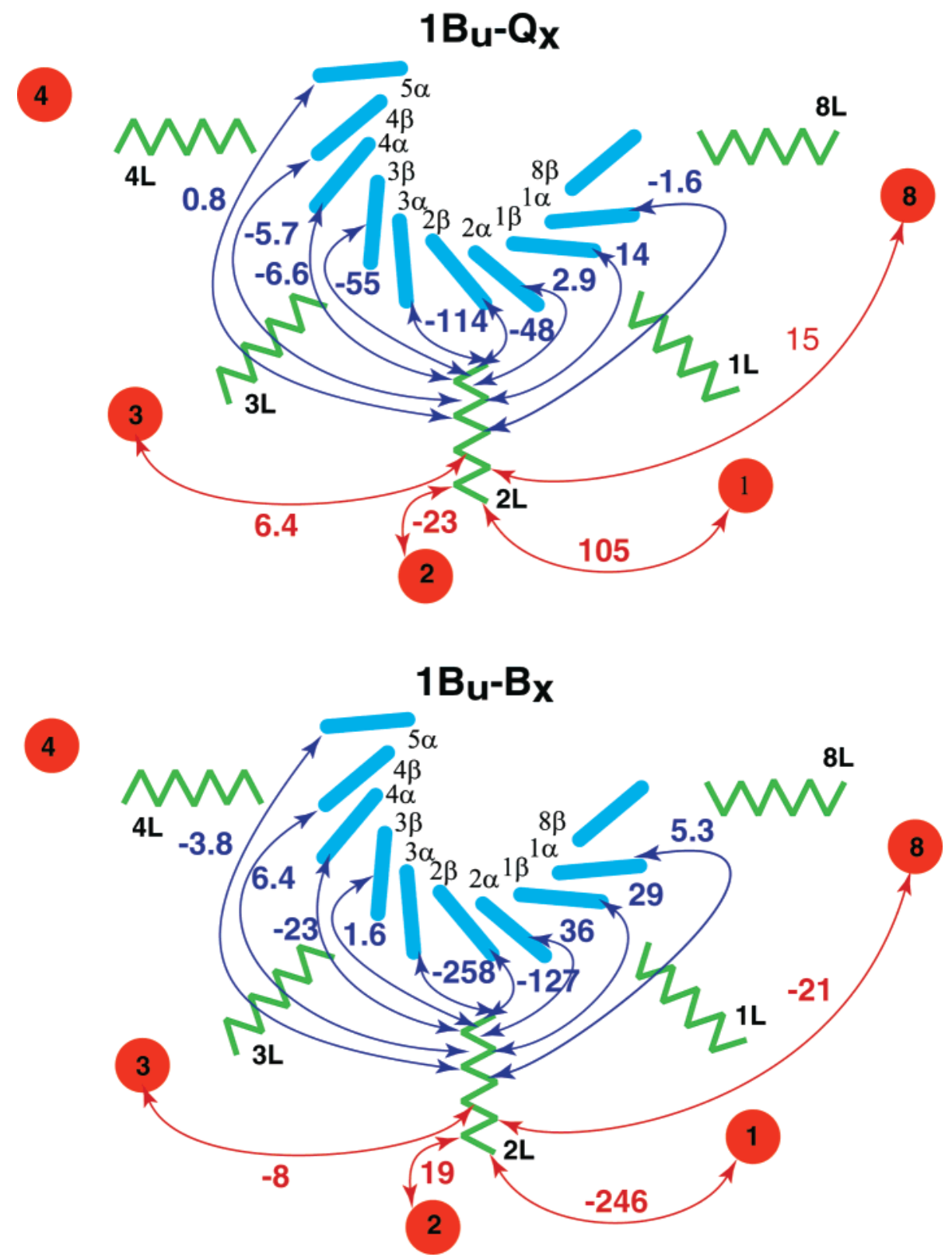

Figure 5. Graphical representation of the calculated excitonic couplings (in $\mathrm{cm}^{-1}$ ) in LH2 of Rs. molischianum shown in Figure 1 in the dielectric medium. These couplings are also given in Table 4.

bands of Lyc aggregates. This is the first report of an effective Hamiltonian for the entire LH2 complex of Rs. molischianum that includes both carotenoid and dielectric medium effects. Using these coupling constants, we have further calculated and discussed the Förster energy transfer rates among different pigments, using the energy flow pathways shown in Figure 2.

Section II briefly describes the CEO method and the Onsager model for solvation energies. The effects of dielectric medium on the Bchl- $a$ and Lyc electronic structure are analyzed in Section III. In Section IV, we outline the methods used for computing the electronic couplings and separating the electrostatic and electron-exchange contributions to the interchromophore interactions. The effective Frenkel exciton Hamiltonian, which takes into account the protein environment effects on the electronic couplings, is presented and analyzed in Section V. Finally, in Section VI, we summarize our results and discuss the energy transfer pathways in LH2.

\section{The CEO and Reaction Field Algorithms}

The numerical CEO-INDO/S procedure for computing electronic structure has been described elsewhere. ${ }^{51,52}$ The ZINDO code was first applied to generate the INDO/S Hamiltonian ${ }^{58-61}$ using experimental geometries of bacteriochlorophylls and carotenoids obtained from crystal structures of the LH2 complex of Rs. molischianum. Hydrogen atoms were added, and their geometries were optimized using semiempirical Austin Model 1 (AM1). ${ }^{62}$ All other atoms were fixed at the crystal structure coordinates. The Hartree-Fock ground-state was calculated by solving the Roothaan-Hall secular selfconsistent field equation iteratively ${ }^{63}$

$$
F C=C \epsilon
$$

where $F$ is the Fock matrix. This equation holds for the closed shell orthogonal basis set considered in this article. Equation 2.1 can be recast in the form $[F(\bar{\rho}), \bar{\rho}]=0$, where the ground- 
TABLE 1. Calculated and Experimental Excitation Energies of $\beta$ B850, $\alpha$ B850, and B800 BChl- $a$ and Lyc Molecules ${ }^{h}$

\begin{tabular}{|c|c|c|c|c|c|}
\hline \multicolumn{6}{|c|}{ Bacteriochlorophyll- $a$} \\
\hline & $\beta \mathrm{B} 850$ & $\alpha \mathrm{B} 850$ & B800 & ${\mathrm{INDO}-\mathrm{CIS}^{c}}^{c}$ & experiment \\
\hline $\mathbf{Q}_{v}(\mathrm{dm})$ & $1.61[771](1.190)$ & 1.59 [781] (1.230) & $1.56[796](1.243)$ & 1.59 [778] (1.789) & $1.6[776]$ \\
\hline (ic) & 1.20 [1035] (1.441) & $1.21[1026](1.456)$ & $1.21[1026](1.464)$ & $1.65[751](2.300)$ & $\left(1.27,{ }^{d} 1.33^{e}\right)$ \\
\hline $\mathbf{Q}_{x}(\mathrm{dm})$ & $2.26[549](0.473)$ & $2.23[557](0.395)$ & $2.25[552](0.351)$ & $2.29[541](0.431)$ & $2.16[575]$ \\
\hline (ic) & $2.13[583](0.194)$ & $2.12[586](0.159)$ & $2.16[575](0.132)$ & $2.25[552](0.128)$ & $(0.685)^{d}$ \\
\hline $\mathbf{B}_{x}(\mathrm{dm})$ & 3.19 [389] (1.194) & $3.14[396](1.213)$ & 3.13 [397] (1.187) & $3.53[351](1.607)$ & $3.17[392]$ \\
\hline (ic) & $3.12[398](1.385)$ & $3.09[402](1.371)$ & $3.11[400](1.332)$ & $3.31[374](0.056)$ & $(\sim 1.11)^{c}$ \\
\hline Tx1 $(\mathrm{dm})$ & $2.96[365](0.073)$ & $2.69[362](0.077)$ & $3.55[350](0.067)$ & & \\
\hline (ic) & $3.40[365](0)$ & 3.34 [372] (0.098) & $3.66[339](0)$ & & \\
\hline $\mathbf{T x} 2(\mathrm{dm})$ & $3.54[350](0.095)$ & $3.33[373](0.127)$ & $3.51[354](0.141)$ & & \\
\hline (ic) & $3.51[354](0.068)$ & $3.48[357](0.055)$ & $3.45[360](0.056)$ & & \\
\hline $\mathbf{T x} 3(\mathrm{dm})$ & $3.68[337](0.355)$ & $3.64[341](0.332)$ & $2.36[526](0.067)$ & & \\
\hline (ic) & $3.53[352](0.117)$ & $3.36[370](0.105)$ & $2.96[420](0.170)$ & & \\
\hline $\mathbf{B}_{y}(\mathrm{dm})$ & $3.42[363](0.883)$ & $3.36[370](0.859)$ & $3.33[313](0.868)$ & $3.51[353](1.115)$ & $3.47[360]$ \\
\hline (ic) & $3.90[318](1.152)$ & $3.87[321](1.170)$ & 3.97 [313] (1.179) & $3.67[338](2.451)$ & $(\sim 0.96)^{c}$ \\
\hline $\mathbf{N}(\mathrm{dm})$ & $4.05[306](0.867)$ & $4.03[308](0.972)$ & $4.09[304](0.956)$ & & \\
\hline (ic) & $4.21[295](0.094)$ & $4.20[296](0.101)$ & $4.29[290](0.112)$ & & \\
\hline \multicolumn{6}{|c|}{ Lycopene } \\
\hline $\mathbf{1 B}_{u}(\mathrm{dm})$ & \multirow{4}{*}{\multicolumn{4}{|c|}{$\begin{array}{l}2.72[457](2.69) \\
2.76[450](2.70) \\
3.65[340](0.11)\end{array}$}} & $2.5[497]$ \\
\hline (ic) & & & & & $(2.71)^{f}$ \\
\hline $\mathbf{2} \mathbf{A}_{g}(\mathrm{dm})$ & & & & & $1.80[762]^{g}$ \\
\hline (ic) & & & & & $3.71[334](0.09)$ \\
\hline
\end{tabular}

${ }^{a} \operatorname{Ref} 50 .{ }^{b} \operatorname{Ref} 73 .{ }^{c} \operatorname{Ref} 48 .{ }^{d} \operatorname{Ref} 74 .{ }^{e} \operatorname{Ref} 36 .{ }^{f} \operatorname{Ref} 75 .{ }^{g} \operatorname{Ref} 91 .{ }^{h} \mathrm{CEO}$ calculations were carried out for an isolated complex ${ }^{a}$ (ic) and in a dielectric medium $(\mathrm{dm})$ with $\epsilon=9$. The experimental transition energies are for Bchl- $a$ monomers in ethyl ether solution ${ }^{b}$. Energies are given in $e V[n m]$. Transition dipole moments (in $e \cdot \AA$ ) are given in round parentheses, and their experimental values for B transitions are estimated from the relative absorbance with possible large margins of error $^{b}$. Molecular labeling is shown in Figures 3, 4 and 5.

TABLE 2. $Q_{y}-Q_{y}$ Bchl- $a$ Interchromophore Couplings $\left(\right.$ in $^{-1} \mathbf{c m}^{-1}$ Calculated for LH2 of Rs. Molischianum

\begin{tabular}{|c|c|c|c|c|c|c|c|c|c|c|c|c|c|c|c|}
\hline & \multirow[t]{2}{*}{$\mathrm{d}$} & \multicolumn{2}{|c|}{$\operatorname{CEO}\left(Q_{y}\right)$} & \multirow{2}{*}{$\frac{\mathrm{a}}{\text { (ic) }}$} & \multirow{2}{*}{$\frac{\mathrm{b}}{\text { (ic) }}$} & \multirow{2}{*}{$\frac{c}{\text { (ic) }}$} & \multirow{2}{*}{$\frac{\mathrm{d}}{\text { (ic) }}$} & \multirow{2}{*}{$\frac{\mathrm{e}}{\text { (ic) }}$} & \multirow{2}{*}{$\frac{\mathrm{f}}{\text { (ic) }}$} & \multirow{2}{*}{$\frac{\mathrm{g}}{\text { (ic) }}$} & \multirow{2}{*}{$\frac{\mathrm{h}}{\text { (ic) }}$} & \multirow{2}{*}{$\frac{\mathrm{i}}{\text { (ic) }}$} & \multirow{2}{*}{$\frac{\mathrm{j}}{\text { (ic) }}$} & \multicolumn{2}{|c|}{$\mathrm{k}$} \\
\hline & & $(\mathrm{dm})$ & (ic) & & & & & & & & & & & (ic) & $(\mathrm{dm})$ \\
\hline $850-850$ & & & & & & & & & & & & & & & \\
\hline $1 \alpha-1 \beta$ & 9.2 & 258 & 363 & 408 & 806 & 339 & 322 & 238 & 367 & 291 & 410 & 394 & 300 & 771 & 622 \\
\hline $2 \alpha-1 \beta$ & 8.9 & 210 & 320 & 366 & 377 & 336 & 288 & 213 & 284 & 273 & 310 & 317 & 233 & 612 & 562 \\
\hline $2 \alpha-1 \beta$ & 18.0 & -67 & -102 & -102 & -152 & & & -46 & -48 & -50 & & & & & \\
\hline $2 \alpha-1 \beta$ & 17.4 & -40 & -63 & -63 & & & & -37 & -37 & -36 & & & & & \\
\hline $1 \alpha-2 \beta$ & 25.6 & 22 & 30 & 31 & & & & & & 12 & & & & & \\
\hline $3 \alpha-1 \beta$ & 25.9 & 17 & 29 & 30 & & & & & & 12 & & & & & \\
\hline $800-850$ & & & & & & & & & & & & & & & \\
\hline $1-1 \alpha$ & 25.5 & 8.2 & 13 & 14 & & 3.8 & -11.3 & -13 & -12 & -12.6 & 7 & -8 & & & \\
\hline $1-1 \beta$ & 20.1 & 25 & 38 & 40 & & 15.7 & 4.8 & 5 & 4 & -3.8 & 6 & -2 & & & \\
\hline $1-2 \alpha$ & 19.2 & -36 & -53 & -52 & & -22.7 & 25.7 & 27 & 27 & 27 & 29 & 16 & & & \\
\hline $\begin{array}{l}1-2 \beta \\
\mathbf{8 0 0}-\mathbf{8 0 0}\end{array}$ & 22.8 & 5.4 & 8.4 & 10 & & 2.9 & 6.1 & 23 & 31 & 12 & 13 & 4 & & & \\
\hline $1-2$ & 22.0 & -19 & -25 & -25 & & -14 & -22 & -27 & -26 & 25 & & -15 & & & \\
\hline $1-3$ & 40.7 & -3.6 & -3.2 & -3.5 & & & & -3 & -3 & & & & & & \\
\hline
\end{tabular}

${ }^{a}$ Ref 50, CEO calculations of splittings in BChls- $a$ dimer spectra. ${ }^{b}$ Ref 47, 48; Semiempirical INDO/CIS calculations of the whole upper ring and further spectral modeling of the results. ${ }^{c}$ Ref 10 , PDA calculations with $\mu^{2}=68 \mathrm{D}^{2} .{ }^{d}$ Ref 10 , PDA calculations with $\mu^{2}=68 \mathrm{D}^{2} .{ }^{e}$ Ref 34 , 39; Ab initio molecular orbital calculations. Transition density cubes approach. ${ }^{f}$ Ref 34, 39; PDA calculations with $\mu=6.13$ D. ${ }^{g}$ Ref 16 , Point monopole approximation based on the SCMO-PPP-CI computations. ${ }^{h}$ Ref 36 , PDA with $\mu^{2}=41 \mathrm{D}^{2} .{ }^{i}$ Ref 17 , Semiempirical QCFF/PI quantum mechanical calculations. ${ }^{j}$ Ref 90 , Modeling the absorption and CD spectra. ${ }^{k}$ Ref 46 , INDO/S/CIS calculations of splittings in BChls- $a$ dimer spectra. ${ }^{l}$ The pigments in the first column are labeled according to Figure 3. The second column shows the $M g-M g$ distance d ( $)$ ). The CEO results in the dielectric medium ( $\mathrm{dm}$ ) (third column) and for an isolated complex (ic) (forth column) (shown in Figure 3 as well) are calculated using Coulomb interaction between transition density matrix elements of monomers. The couplings computed from splittings in the dimer spectra are shown in the fifth column (a). The table also summarizes couplings for $Q_{y}$ band reported in the literature for Rs. molischianum (Columns a-c), and Rps. acidophila (Columns d-k).]

state density matrix $\bar{\rho}^{64,65}$ is related to the molecular orbital expansion coefficients $C$ through

$$
\bar{\rho}_{n m}=2 \sum_{a}^{N} C_{n a} C_{m a}
$$

where $N$ is the number of electrons in the aggregate. The Fock matrix for the isolated aggregate is

$$
F^{0}(\bar{\rho})=t+V(\bar{\rho})
$$

where the first term is the core-Hamiltonian describing the kinetic energy and nuclear attraction of an electron

$$
\begin{aligned}
t_{n m}=\left\langle n\left|-\frac{1}{2} \nabla_{1}^{2}-\sum_{\mathrm{A}} \frac{Z_{\mathrm{A}}}{\left|\boldsymbol{r}_{1}-\boldsymbol{R}_{\mathrm{A}}\right|}\right| m\right\rangle \equiv \\
\quad \int d \boldsymbol{r}_{1} \chi_{n}^{*}(1)\left(-\frac{1}{2} \nabla_{1}^{2}-\sum_{\mathrm{A}} \frac{Z_{\mathrm{A}}}{\left|\boldsymbol{r}_{1}-\boldsymbol{R}_{\mathrm{A}}\right|}\right) \chi_{m}(1)
\end{aligned}
$$

$\mathbf{R}_{\mathrm{A}}$ is the nuclear coordinate of atom $A$ and $\left\{\chi_{\alpha}\right\}$ is the atomic 
TABLE 3. $B_{x}-B_{x}$ Bchl- $a$ Interchromophore Couplings (in $\mathrm{cm}^{-1}$ ) Calculated for LH2 of Rs. Molischianum ${ }^{b}$

\begin{tabular}{lccc}
\hline & \multicolumn{3}{c}{ CEO $\left(B_{x}\right)$} \\
\cline { 2 - 4 } & $(\mathrm{dm})$ & (ic) & ${\text { (ic })^{a}}$ \\
\hline B850-B850 & 248 & 344 & 367 \\
$1 \alpha-1 \beta$ & 343 & 364 & 369 \\
$2 \alpha-1 \beta$ & 42 & 60 & 61 \\
$2 \alpha-1 \beta$ & 33 & 58 & 58 \\
$2 \alpha-1 \beta$ & 14 & 21 & 23 \\
$1 \alpha-2 \beta$ & 18 & 20 & 20 \\
$3 \alpha-1 \beta$ & 6.2 & 5.9 & 6.1 \\
B800-B850 & 24 & 32 & 32 \\
$1-1 \alpha$ & -11 & 5.4 & 6.9 \\
$1-1 \beta$ & -15 & -23 & -23 \\
$1-2 \alpha$ & 18 & & \\
$1-2 \beta$ & 3.3 & 2.0 & 7.0 \\
B800-B800 & & 2.2 & 2.3 \\
$1-2$ & & &
\end{tabular}

${ }^{a}$ Ref. 50 CEO calculations of splittings in BChls- $a$ dimer spectra ${ }^{b}$ The pigments in the first column are labeled according to Figure 3. The CEO results in the dielectric medium (dm) (second column) and for an isolated complex (ic) (third column) (shown in Figure 3 as well) are calculated using Coulomb interaction between diagonal transition density matrix elements of monomers. The couplings computed from splittings in the dimer spectra are shown in the forth column.

basis set. The second term represents electron-electron Coulomb interactions, with the matrix elements

$$
V(\bar{\rho})_{m n}=\sum_{k, l}^{K} \bar{\rho}_{k l}\left[\langle m k \mid n l\rangle-\frac{1}{2}\langle m n \mid k l\rangle\right]
$$

$K$ being the basis set size, and

$$
\langle n m \mid k l\rangle=\int d \mathbf{r}_{1} d \mathbf{r}_{2} \chi_{n}^{*}(1) \chi_{m}^{*}(2) \frac{1}{r_{12}} \chi_{k}(1) \chi_{l}(2)
$$

are the two-electron integrals.

The INDO approximation ${ }^{59}$ limits the basis set to valence orbitals of Slater type. Exchange terms in the two-electron interaction are only permitted among orbitals centered on the same atom. The tetradic matrix $\langle n m \mid k l\rangle$ thus becomes blockdiagonal dyadic, i.e., $\langle n m \mid k l\rangle=\langle n m \mid n m\rangle \delta_{n k} \delta_{m l}$ when the atomic orbitals $n$ and $m$ belong to different atoms. This approximation allows us to limit the number and store all computed Coulomb matrix elements in memory instead of recalculating them when needed, as is done in ab initio approaches, making semiempitical techniques significantly easier and faster. The INDO/S Hamiltonian parameters are given in [59-61].

Finally, the CEO procedure ${ }^{52,51}$ was applied to compute the linear-absorption spectra and the relevant transition density matrices (denoted electronic normal modes $\xi_{v}$ ), which connect the optical response with the underlying electronic motions. Each mode is a $K \times K$ matrix representing the electronic transition between the ground state $|g\rangle$ and an electronically excited state $|v\rangle$. Its matrix elements are given by

$$
\left(\xi_{v}\right)_{m n}=\left\langle\nu\left|c_{m}^{+} c_{n}\right| g\right\rangle
$$

where $c_{m}^{+}\left(c_{m}\right)$ are creation (annihilation) operators of an electron at the $m$ th atomic orbital, and $\mid g(|v\rangle)$ is the ground (excited) state many-electron wave function. $\xi_{v}$ represents collective correlated motions of electrons and holes and carry substantially less information than the many-electron eigenstates but more than required for calculating all spectroscopic observables such as molecular polarizabilities. The diagonal element $\left(\xi_{v}\right)_{n n}$ is the net charge induced on the $n$th atomic orbital by an external field with frequency $\Omega_{v}$, whereas $\left(\xi_{v}\right)_{m n} n \neq m$ is the dynamical bondorder (coherence) representing the joint amplitude for finding an electron on orbital $m$ and a hole on $n$. The modes are computed directly as eigenmodes of the linearized timedependent Hartree-Fock (TDHF) equations of motion for the density matrix driven by the external field, totally avoiding the explicit calculation of many-electron excited-state wave functions

$$
L \xi_{v}=\Omega_{v} \xi_{v}, L \xi_{v}^{+}=-\Omega_{v} \xi_{v}^{+}, v=1, \ldots, N \times K
$$

The eigenfrequencies, $\Omega_{v}$, provide the optical transition frequencies. ${ }^{52,51} \mathrm{~L}$ is a linear operator in Liouville space (i.e., superoperator $)^{52,51}$ given by

$$
L \xi=[F(\bar{\rho}), \xi]+[V(\xi), \bar{\rho}]
$$

where $\xi$ is an arbitrary $K \times K$ matrix.

The numerical effort involved in computing the eigenvalues, $\Omega_{v}$, and eigenvectors, $\xi_{v}$, is greatly reduced by using the oblique Lanczos algorithm. ${ }^{66,67}$ Transition dipole moments $\vec{\mu}_{v}=\operatorname{Tr}\left(\vec{\mu} \xi_{v}\right)$ are then calculated using the dipole moment operator $\vec{\mu}=$ $\sum_{n m} \vec{\mu}_{n m} c_{m}^{+} c_{n}$, and

$$
f_{v}=2 \Omega_{v} \vec{\mu}_{v}^{2}
$$

is the oscillator strength of the $g$ to $v$ transition.

To include the effects of the surrounding media, we have used the Self-Consistent Reaction Field (SCRF) approach, ${ }^{48,68,69}$ whereby the interaction energy between a solute and the surrounding medium is added to the HF energy of an isolated molecule, and the total energy of the system is then minimized self-consistently. Because the solute is electrically neutral in our case, only the dipolar interactions contribute to the solvation energy. The Fock operator $F_{m n}^{0}$ is then modified by adding the response of a dielectric medium, resulting in

$$
F_{m n}=F_{m n}^{0}-\frac{\epsilon-1}{2 \epsilon+1} \frac{\vec{\mu}_{g} \cdot \vec{\mu}_{m n}}{a_{o}^{3}}
$$

where $F_{m n}^{0}$ is the isolated complex Fock operator, $\vec{\mu}_{g}$ is the ground-state dipole moment, $\epsilon$ is the dielectric constant, and $a_{o}$ is a cavity radius. The second term in eq 2.11 (Onsager dipolar term) has been derived, ${ }^{68,69}$ assuming that the solute is separated from the solvent by a sphere of radius $a_{o}$.

Onsager's SCRF is the simplest method for taking dielectric medium effects into account. Even though spherical cavity is a crude approximation for the flat Bchl- $a$ or linear Lyc molecules, the predicted trends usually agree well with experiment and with the results of much more sophisticated and expensive methods. ${ }^{68,69}$ Using eq 2.11, we calculated Bchl- $a$ and Lyc monomers assuming a dielectric constant $\epsilon=9$, suggested in (70) (the index of refraction was estimated to be 1.6 in (71)) to describe the effects of the protein environment on excited-state energies and the relevant transition densities. These densities were then used to calculate the Coulomb couplings. The same approach was used in INDO/S/CIS study ${ }^{48}$ of dielectric medium effect on absorption spectra of Bchls aggregates. The cavity radii were calculated with the Gaussian 98 package $^{72}$ at the ab initio $6-31+\mathrm{G}^{*}$ HF level using the keyword Volume, which provides a reasonable estimate for a radius of the Onsager solvent reaction field model. This gives 5.6 $\AA$ and $7.1 \AA$ radii for Bchl- $a$ and Lyc molecules, respectively. 
TABLE 4. Bchl-Lyc Interchromophore Couplings (in $\mathrm{cm}^{-1}$ ) Calculated for LH2 of Rs. Molischianum ${ }^{g}$

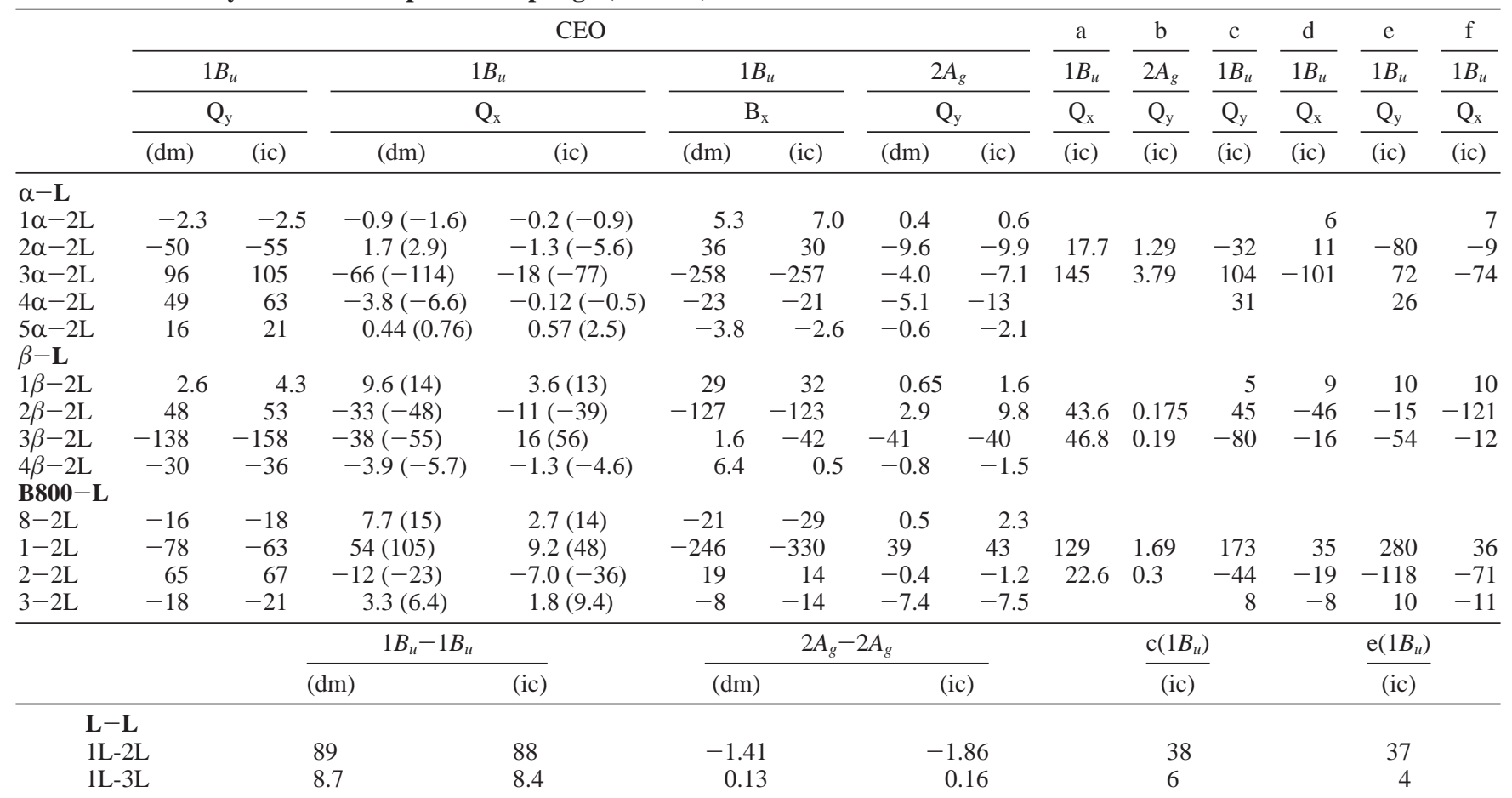

${ }^{a, b} \operatorname{Ref} 49$, Coulomb interaction between transition densities based on PPP/CIS method. ${ }^{c, d}$ Ref 34, 39; Ab initio molecular orbital calculations. Transition density cubes approach. ${ }^{e}{ }^{f} \operatorname{Ref} 34,39$; PDA calculations with $\mu_{Q_{y}}=6.39 \mathrm{D}, \mu_{Q_{x}}=3.29 \mathrm{D}, \mu_{1 B_{u}}=13 \mathrm{D}$. ${ }^{g}$ The pigments in the first column are labeled according to Figures 4 and 5. The CEO couplings between $1 B_{u}-Q_{y}$ (second column), $1 B_{u}-Q_{x}$ (third column), and $1 B_{u}-B_{x}$ (forth column) transitions (shown in Figures 4 and 5 as well) are calculated using Coulomb interaction between diagonal transition density matrix elements of monomers in the dielectric medium (dm) and for an isolated complex (ic). The couplings involving $Q_{x}$ transition rescaled with factors $\left(\mu_{\text {exp }} / \mu_{\text {calc }}\right)_{Q_{x}}$ are shown in round parenthesis. These factors are 1.73, 1.45, 1.95 in (dm) and 4.3, 3.5, and 5.2 in (ic) for $\alpha, \beta$ B850, and B800 chromophores, respectively (see Table 1). The table also summarizes couplings for $1 B_{u}-Q_{y}$ and $1 B_{u}-Q_{x}$ states reported in the literature for Rs. molischianum (column a) and Rps. acidophila (columns b-d).

The Onsager SCRF model makes several approximations: spherical cavity, uniform dielectric medium, and point dipole approximation (PDA). We have used it for single chromophore (Bchl or Lyc) calculations. The model reproduces solvation effects very well and the calculated absorption spectrum of single Bchl in a dielectric medium is in excellent agreement with experiment and is significantly better then that calculated for an isolated complex. We have used the PDA in the SCRF model, but not for the sake of computing the intermolecular couplings. The SCRF takes into account solvent effects that are highly averaged and only weakly perturb the electronic structure. The PDA is then adequate. Intermolecular couplings are stronger, and the spectra are much more sensitive to their detailed form. The PDA may not be justified for these couplings in aggregates where the chromophore sizes are comparable to their distances. We have, therefore, empolyed the SCRF approach to compute transition densities of single chromophores and then used these charge distributions to compute coulomb interchromophore couplings without invoking the PDA.

\section{Dielectric Effects in the Linear Absorption of Bchl- $a$ and Lyc Monomers}

The calculated properties of individual Bchl- $a$, both isolated and in a dielectric medium are summarized in Table 1. Columns 2-4 show the electronic spectra of the various Bchl- $a$ monomers ( $\alpha$ and $\beta$ B850 and B800 molecules). Despite being the same Bchl- $a$ species, the crystal structure geometries are slightly different, which affects the spectra. For comparison, the table also includes the INDO/S/CIS calculations of Bchl- $a$ monomers (isolated complex and dielectric medium) reported in [48]. These computations used the same crystal structure geometry of $R s$. molischianum. The table also contains experimental data for Bchl- $a$ monomers in solution. ${ }^{73-75}$ Detailed analysis of the isolated complex CEO linear absorption spectra and the relevant transition densities of BChl- $a$ was given in [50]. Below, we solely focus on the protein solvation effects.

The solvent significantly affects the ground state. Its dipole moment $\mu_{g}$ increases from 6.8 to $20.5 \mathrm{D}$ (similar enhancement from 7.3 to $21 \mathrm{D}$ was reported in INDO/S/CIS calculations ${ }^{48}$ because both approaches use the same ground state). Furthermore, the protein environment has a considerable impact on the excited states. We use the standard labeling of active optical peaks for porphyrin-like systems $(\mathrm{Q}, \mathrm{B}, \mathrm{N})$. Additional transitions are denoted Tx1-Tx3. We start with the $\beta$ B850 linear absorption spectrum shown in column 2 of Table 1 .

The lowest $Q_{y}$ transition with a strong oscillator strength plays a major role in the light absorption function of the antenna. The computed $1.2 \mathrm{eV}$ frequency of the isolated complex underestimates the experimental $(1.6 \mathrm{eV})$ value. However, the protein leads to a significant blue shift of frequency to 1.61 $\mathrm{eV}$, which matches the experiment. This is contrary to the INDO/S/CIS result, which shows only a small solvent induced red-shift. This may be attributed to the different approximations for the excited many-electron wave function used in INDO/S/ CIS, which is based on CIS, and the CEO based on the TimeDependent Hartree-Fock (TDHF) anzatz. Both CEO and INDO/S/CIS consistently predict $15-20 \%$ solvent reduction of the $Q_{y}$ transition dipole. The CEO transition dipoles agree well with experiment (further rescaling of the electronic couplings to match experiment is no longer necessary). In contrast, INDO/ S/CIS overestimates experiment (this is usually the case in CIS 
computations) and further rescaling of the couplings may be necessary.

The isolated complex energy of the next $Q_{x}$ state $(2.13 \mathrm{eV})$ matches the experiment $(2.16 \mathrm{eV})$. Similar to the INDO/S/CIS calculations, the protein environment induces a small blue shift to $2.26 \mathrm{eV}$. However, the $Q_{x}$ transition dipole drastically changes in the solvent. $Q_{x}$ is only weakly allowed in linear absorption of the isolated complex. Dielectric medium increases its transition dipole by factor 2.5 (3.3 in INDO/S/CIS). This could be attributed to oscillator strength borrowing from the strong neighboring $Q_{y}$ and $B_{x}$ transitions whose intensities are decreased in solution. Although the $Q_{x}$ transition dipole in the solvent is much closer to experiment than the isolated complex value, it is still weaker by factor 1.5 , which should be applied for rescaling the couplings involving $Q_{x}$ transition. This difference may be due to additional vibronic coupling of $Q_{x}$ to the states with large oscillator strengths.

The following strong $B_{x}$ and $B_{y}$ peaks represent components of the Soret transition. Similar to $Q_{x}$, the protein environment only weakly shifts the following $B_{x}$ excited-state energy to the blue. Both isolated complex $(3.12 \mathrm{eV})$ and dielectric medium $(3.19 \mathrm{eV})$ Soret frequencies are in excellent agreement with experiment $(3.17 \mathrm{eV})$. INDO/S/CIS calculations overestimate the $B_{x}$ frequencies and predict a more pronounced blue shift. Similar to $Q_{y}$, the solvent decreases the intensity of $B_{x}(15 \%$ reduction of the transition dipole). However, accurate measurements of the Soret oscillator strength are not available. The $B_{x}$ transition dipole could only be crudely estimated from the relative peak intensities in the absorption spectrum. ${ }^{73}$ We expect the CEO to adequately reproduce the $B_{x}$ transition dipole, as in the case of porphins. ${ }^{57,76}$ Indeed, the calculated values agree with experimental estimates (see Table 1).

The $B_{y}$ transition frequency $(3.90 \mathrm{eV})$ in the isolated complex is significantly higher than experiment $(3.47 \mathrm{eV})$. However, the solvent induces a strong red-shift of this transition to $3.42 \mathrm{eV}$, bringing it to an excellent agreement with experiment. A similar solvent-induced red shift of $B_{y}$, which brings it closer to experiment, has been reported in INDO/S/CIS calculations. Both CEO and INDO/S/CIS predict solvent reduction of the $B_{y}$ transition dipole moment ( $23 \%$ and $55 \%$, respectively). The CEO transition dipole agrees with a crude experimental estimate (Table 1). The high frequency $\mathrm{N}$ transition is weak for an isolated complex. The dielectric medium dramatically enhances its transition dipole by a factor of 9 and induces a $0.2 \mathrm{eV}$ redshift. The solvent further shifts the charge transfer transitions Tx1-Tx3 either to the red or to the blue, and furthermore makes them weakly allowed. Very similar spectra and analogous protein environment effects are observed in the $\alpha B 850$ and B800 molecules (columns 3 and 4 in Table 1).

We next turn to the linear absorption of Lycopene. Even though the carotenoid is nearly symmetric, the dielectric medium still increases the ground-state dipole moment from 1.7 to 3.1 D. However, both frequency and transition dipole of the main absorption peak corresponding to $1 B_{u}$ transition are barely affected by solvent (see Table 1). Compared to experiment, the calculated frequency is blue-shifted by $0.2 \mathrm{eV}$, but its transition dipole is in excellent agreement. Our calculations do not predict the correct $2 A_{g}$ state energy, which is known to be the case for TDHF or CIS calculations. Reproducing the correct order of the states $\left(2 A_{g}\right.$ lower than $\left.1 B_{u}\right)$ requires expensive calculations including higher order electronic correlations. ${ }^{77}$ The Lyc crystal structure geometry is not fully symmetric. This results in a very weak transition dipole of the $2 A_{g}$ state. The protein environment further breaks the symmetry, slightly increasing the $2 A_{g}$ transi- tion dipole (see Table 1), leading to the finite radiative lifetime of this state. ${ }^{78}$

In concluding this section, we note that the linear absorption spectra calculated with the Onsager SCRF model are in much better agreement with experimental spectra in solution than those of the calculated isolated-complex spectra. Both excited state frequencies and their transition dipoles closely match the available experimental values. These results establish a firm basis for the following investigation of interchromophore electronic interactions.

\section{Spectroscopic vs. Coulombic Calculation of Electronic Couplings}

The electronic excitations of an assembly of two-level chromophores can be described by the Frenkel exciton model ${ }^{79-81}$

$$
H=\sum_{n} \Omega_{n} B_{n}^{+} B_{n}+\sum_{n \neq m} J_{n m} B_{m}^{+} B_{n}
$$

Here, $B_{m}\left(B_{m}^{+}\right)$is the annihilation (creation) operator of an excitation localized on the $m$ th chromophore, and $\Omega_{n}$ represents the transition energy from the ground state to the excited state on the $n$th chromophore. The interaction between chromophores is described by the hopping parameters $J_{n m}$. When the chromophores are spatially well separated, their interaction is purely electrostatic. However, at closer separations, intermolecular electron exchange processes become allowed, ${ }^{55}$ making additional contributions to the chromophore couplings. These distinct Coulombic and electron exchange interactions ${ }^{55}$ are known as the Förster and the Dexter couplings, respectively.

To separate these interactions we have employed two different methods for computing the couplings. Both calculations used the actual crystal structure geometries for segments of the LH2 complex. The spectroscopic approach is based on computing the Davydov splitting $79,80,82$ in the dimer spectra. This method produces accurate results when the chromophores are identical or nearly identical and when the contribution from other electronic states to the dimeric splitting is negligible, which is the case provided the other states are well separated (for details see (50)). In particular, for identical chromophores A and B we have $J_{A B}=|\Delta \epsilon| / 2$, where $\Delta \epsilon$ is the Davydov splitting between a pair of dimer states. The coupling computed in this fashion includes both the Förster and the Dexter contributions. In the previous article, ${ }^{50}$ we applied this approach to compute couplings among nearly identical Bchls- $a$ of the LH2 complex.

Alternatively, the purely electrostatic coupling between two monomers may be represented as Coulomb interaction between the diagonal elements of their transition density matrices

$$
J_{A B}=\sum_{n \in A, m \in B} V_{n m}\left(\xi_{v}^{A}\right)_{n n}\left(\xi_{v}{ }^{B}\right)_{m m}
$$

where $\left(\xi_{v}^{A}\right)_{n m}$ and $\left(\xi_{v}^{B}\right)_{m m}$ are the diagonal matrix elements of the electronic modes of the monomers $\mathrm{A}$ and $\mathrm{B}$, and $V_{n m}$ is a Coulomb interaction between charges $\left(\xi_{v}^{A}\right)_{n n}$ and $\left(\xi_{v}^{B}\right)_{m m}$. To obtain couplings among Bchl- $a$ and Lyc using eq 4.2, we first calculated the electronic modes of the monomers $\left(\left(\xi_{v}^{A}\right)_{n n}\right.$ and $\left.\left(\xi_{v}^{B}\right)_{m m}\right)$ and then sorted out the INDO/S Coulomb two-electron integrals $V_{n m}=\langle m n \mid m n\rangle$ relevant for the $\mathrm{AB}$ dimer pair.

When electron exchange is negligible, the spectroscopic and coulomb methods give identical couplings (note that using Coulomb elements $V_{n m}$ other then INDO/S parametrization (e.g., actual electrostatic interaction or $a b$ initio matrix elements) would be inconsistent and produce different spectroscopic and coulomb couplings). A similar approach (denoted the two-level 
model) was applied for estimating interchromophore interactions in photoluminescent polymer aggregates. ${ }^{83}$ Although this method is analogous to the point monopole approximation technique used in [16] for computing effective Frenkel Hamiltonian in LH2 of Rps. acidophila, it does not suffer from uncertainty in the charge reduction scheme such as Milliken population analysis, because both coulomb interaction elements and transition densities are given in the same basis set, consistent with the computational algorithm. We further note that the Coulomb method is numerically more attractive than the spectroscopic approach because it only requires the calculation of monomer spectra; dimer spectra are not needed. In the case of a finite electron exchange interaction, the difference between couplings computed with these two approaches gives the Dexter contribution. In this article, we have separated the two contributions using calculations performed on the isolated complex. The incorporation of solvation effects in these estimates will require a much more intensive effort.

The spectroscopic technique that computes the overall interaction, is not sufficiently accurate for calculating couplings between two energetically well separated states because the contributions from other electronic states may not be neglected. On the other hand, the Coulomb approach only takes the electrostatic part of the interaction into account but may be applied for arbitrary pair of electronic states of any two molecules. The two methods are thus complimentary, and combining them allows to pinpoint the origin of interchromophore interactions. For example, computing Bchl-Lyc interaction is only possible with the second method. These chromophores are very different and the numerical accuracy of specroscopic procedure is low.

\section{Frenkel Exciton Hamiltonian in a Dielectric Medium}

Bchl-Bchl interaction. We first compare the spectroscopic and coulomb calculations in order to separate the Förster and the Dexter contributions to the couplings. Column 4 and column a in Table 2 show couplings for Bchl- $a Q_{y}$ band calculated with both methods for an isolated complex. We expect the weak electron exchange interaction to show up only for closely lying molecules such as $\alpha \beta$ intra- and inter dimers. Indeed, comparison of Coulombic and spectroscopic couplings for these pairs (363 $\mathrm{cm}^{-1}$ vs $408 \mathrm{~cm}^{-1}$, and $320 \mathrm{~cm}^{-1}$ vs $366 \mathrm{~cm}^{-1}$ ) shows that electron exchange makes a $45 \mathrm{~cm}^{-1}$ contribution to the interaction in these dimers. Very similar 45 and $40 \mathrm{~cm}^{-1}$ values for intra- and inter- B50 dimers were reported in [39]. On the other hand, molecules in all other Bchl- $a$ pairs are well separated and therefore the Dexter contribution to their couplings is negligible (differences of up to $1 \mathrm{~cm}^{-1}$ may be attributed to the effects of higher lying electronic states and numerical errors).

Similar trends are observed for Bchl- $a B_{x}$ band couplings computed with these two approaches and shown in columns 3 and 4 of Table 3. However, the electron exchange contribution ( $23 \mathrm{~cm}^{-1}$ and $5 \mathrm{~cm}^{-1}$ for $\alpha \beta$ intra- and inter dimers, respectively) is considerably reduced compared to the $Q_{y}$ interactions. Again, the Dexter contribution is negligible for all other Bchl- $a$ pairs which are well separated in space.

We next turn to discussing the protein environment effects on electronic intermolecular interactions. Column 3 in Table 2 shows $Q_{y}$ couplings among different Bchl- $a$ molecules calculated with the coulomb method in a dielectric medium. Comparison with the isolated complex results (column 4) shows significant ( $\sim 30 \%$ ) decrease of the coupling strength from $363 \mathrm{~cm}^{-1}$ to $258 \mathrm{~cm}^{-1}$ due to the environment. This number is in remarkable agreement with a recent observation of quantum beats between exciton states of the B820 dimeric subunit, which gave the exciton splitting of about $500 \mathrm{~cm}^{-1}$ (i.e., coupling of 250 $\left.\mathrm{cm}^{-1}\right) .{ }^{84,85}$ Very close estimates for these couplings were also obtained from the analysis of the relative difference absorption of the B820 subunit and B850 antenna. ${ }^{86}$ This also agrees with the results reported in (46) (column $\mathrm{k}$ in Table 2) where the nearest to the Bchl- $a$ chromophores proteins were explicitly taken into INDO/S/CIS calculations to model the dielectric medium effect. This effect could be attributed in part to the reduction of the $Q_{y}$ transition dipole due to solvent: the $\sim 1.2$ decrease of the transition dipole roughly leads to the reduction of couplings by factor $1.2^{2}=1.44$. However, this quadratic dependence is not exact because the PDA is not applicable for this aggregate.

A similar decrease of coupling strength is generally observed for the Bchl- $a B_{x}$ band (columns 3 and 4 of Table 3). It is interesting to note that $\alpha \beta$ intra-dimer interaction is significantly reduced, whereas $\alpha \beta$ inter-dimer coupling only weakly depends on solvation. In fact, all B800-B800 and some B850-B800 interactions even increase in a dielectric medium. This again points to the failure of PDA and to the collective effect of spacecharge redistribution on interchromophore interactions.

The effective Hamiltonian parameters calculated in the protein environment for Bchl-Bchl $Q_{y}$ and $B_{x}$ bands are displayed in Figure 3. The computed Bchl- $a Q_{y}$ and $B_{x}$ transition dipoles agree well with experiment, requiring no further rescaling or modification. We note that $Q_{y}$ couplings are well within the range of parameters reported for $\mathrm{LH} 2$ systems (columns $\mathrm{b}-\mathrm{k}$ of Table 2), with the exception of B850-B800 interactions, which systematically exceed other computational results.

Lyc-Bchl interaction. As stated earlier, the spectroscopic approach is inapplicable for computing bacteriochlorophylllycopene interactions. Table 4 compares the isolated complex and dielectric medium results computed with the Coulombic method. Electronic interactions between $1 B_{u}$ Lyc state and three low energy states $\left(Q_{y}, Q_{x}\right.$, and $\left.B_{x}\right)$ of Bchl- $a$ were calculated. Each Lyc molecule has a fairly strong interaction with half of Bchls- $a$ in the upper B850 and lower B800 circles. These $1 B_{u}-$ $Q_{y}$ couplings are displayed in Table 2, columns 2 and 3, the largest values exceed hundred wavenumbers. The dielectric medium only makes a weak effect on their magnitudes, resulting in a slight decrease of interactions with the B850 ring and an increased coupling to the B800 ring. Our calculated parameters correlate well with the results reported in refs 34,39 for the isolated Rps. acidophila complex (Table 2, columns c and d).

$1 B_{u}-Q_{x}$ couplings shown in columns 4 and 5 of Table 4 require a more careful analysis. Their isolated-complex values are very small because the calculated transition dipole of $Q_{x}$ is much weaker compared to experiment (see Section III). This discrepancy may be corrected by introducing rescaling factors which are the ratios of experimental to computed transition dipoles. The couplings rescaled by factor $\left(\mu_{\text {exp }} / \mu_{\text {calc }}\right)_{Q_{x}}$ are shown in parentheses of column 5 (the lycopene transition dipole need no rescaling). However, these rescaling factors are very large (4-5), and we do not believe these calculations to be accurate because the isolated-complex transition densities distribution is very different from that in a dielectric medium. In contrast, the $Q_{x}$ transition dipole in the protein environment is much larger and comparable with experiment. Consequently, the calculated couplings are significantly higher. The $Q_{x}$ transition density is now more realistic and could be roughly represented as a superposition of the isolated complex $Q_{x}, Q_{y}$, and $B_{x}$ transition densities (see Section III). Note that we do not have such strong solvent effect on the charge distribution in the transition densities 
of the intense $Q_{y}$ and $B_{x}$ states. However, the computed $Q_{x}$ transition dipole is still weaker than experiment, and smaller rescaling factors $(1.4-2)$ need to be introduced. Rescaled couplings shown in parentheses (see column 4 of Table 4) are now reasonably accurate. It is interesting to note the significant differences between the isolated complex and dielectric medium rescaled parameters shown in columns 4 and 5 (parenthesis). This points out to the necessity of properly taking into account solvent effects for the accurate determination of Lyc interactions with $Q_{x}$ band of Bchls- $a$. Our calculated parameters are very similar to the isolated complex results reported in (49) for $R s$. molischianum (Table 4, columns a and b). PPP/CIS calculations employed in (49) generally overestimate the transition dipole magnitudes. They resulted in the right dipole of $Q_{x}$ transition, whereas the $Q_{y}$ transition dipole was overestimated by a factor of $\sim 2$.

$1 B_{u}-B_{x}$ couplings are shown in columns 6 and 7 of Table 4. It is interesting to note the extremely strong interaction (up to $330 \mathrm{~cm}^{-1}$ ) between Lyc and one Bchl- $a$ from the upper ring $(3 \alpha-2 L)$ and one Bchl- $a$ from the lower ring $(1-2 L)$. The carotenoid strongly bridges the B850 and B800 rings in this energy region. This energy transfer pathway from the Soret states of Bchl- $a$ to the $1 B_{u}$ state of Lyc should be extremely efficient and may effectively compete with the internal conversion on the individual Bchls- $a$, in the same way the intermolecular $1 B_{u}-Q_{x}$ channel successfully competes with $1 B_{u}-2 A_{g}$ relaxation on the carotenoid. Further experimental and theoretical studies will be required to address this issue. Protein environment effects vary: some dimer pairs such as $3 \beta-2 L$ are strongly affected, others only show minor influence.

Even though the $2 A_{g}-Q_{y}$ couplings shown in columns 8 and 9 of Table 4 are smaller than the corresponding $1 B_{u}$ couplings, they can still be as large as $40 \mathrm{~cm}^{-1}$. Because the $2 A_{g}$ state has a very weak transition dipole, quadrupole and higher multipole representations have been employed to compute the $2 A_{g}-Q_{y}$ couplings. ${ }^{49}$ However, interaction between transition densities would be the most accurate prescription for computing these parameters. Compared to PPP/CI calculations reported in (49) our $2 A_{g}-Q_{y}$ results (Table 4 , columns 8 and 9) significantly exceed the PPP/CI couplings, whereas the $1 B_{u}-Q_{x}$ parameters are very similar in both approaches. This may be attributed to two factors. First, the INDO/S Hamiltonian, which includes both $\pi$ and $\sigma$ orbitals, is better than the PPP Hamiltonian, which only has $\pi$ basis functions. We found that both $\sigma$ bonding and hydrogen atoms make significant contribution to the $2 A_{g}-Q_{y}$ interaction. Second, unlike our calculations in (49), the $2 A_{g}$ state was treated as completely forbidden in linear absorption.

Finally, a comparison of the dielectric medium and isolated complex results (Table 4, columns 8 and 9) shows that the protein environment generally reduces the interaction. This may not be solely attributed to the decrease of the $Q_{y}$ transition dipole by the solvent reaction field, but rather reflects a more complex redistribution of transition charge densities.

The resulting effective Hamiltonian parameters calculated in the protein environment for Lyc-Bchl $Q_{y}, Q_{x}$, and $B_{x}$ bands are displayed in Figures 4 and 5 . The $1 B_{u}-Q_{y}$ and $1 B_{u}-B_{x}$ values need no further rescaling or modification because the computed Bchl- $a Q_{y}$ and $B_{x}$ and Lyc $1 B_{u}$ transition dipole magnitudes agree well with experiment. $1 B_{u}-Q_{x}$ couplings are rescaled by the $\left(\mu_{\text {exp }} / \mu_{\text {calc }}\right)_{Q_{x}}$ ratios given in caption to Table 4 .

Lyc-Lyc interaction. The carotenoid couplings are given at the bottom of Table 4. Because these molecules are well separated, both Coulombic (Table 4, column 3) and spectroscopic (not shown) calculations give identical couplings. As expected, the dielectric medium has a very weak effect on intermolecular interactions (compare Table 4, columns 2 and 3,4 and 5). Our calculated $2 A_{g}-2 A_{g}$ couplings are very weak, whereas $1 B_{u}-1 B_{u}$ values are large and significantly exceed the inter-carotenoids couplings reported in $(34,39)$ for Rps. acidophila. This may be attributed either to geometry differences between Rs. molischianum from Rps. acidophila or to different levels of computation. The effective $1 B_{u}-1 B_{u}$ Lyc Hamiltonian parameters calculated in the protein environment are also shown in Figures 4 and 5.

\section{Discussion}

Low frequency $Q_{y}$ near-infrared excitations constitute the major energy flow path in the LH2 antenna, which is triggered by photons absorbed by higher frequency (visible and UV) Bchls- $a$ transitions and carotenoids $1 B_{u}$ state. Joint theoretical and experimental input is required for successful modeling of the photophysical function of antennae. ${ }^{41,42,10}$ Information on energetic disorder, protein relaxation and energy transfer time scales, spectral overlaps, etc. may be extracted from femtosecond nonlinear optical studies, ${ }^{18-32}$ and computing of the necessary electronic coupling constants is the subject of an intensive theoretical effort. ${ }^{16,34,39,48-50}$

Our electronic couplings for an isolated complex and in the dielectric medium calculated using a unified CEO framework represent one of the most accurate and complete effective Frenkel exciton Hamiltonian of LH2 reported to date (Tables 2, 3, and 4 and Figures 3, 4, and 5). These parameters could be employed to estimate B800-B800, B800-B850, Lyc-B850, and Lyc-B800 energy transfer time scales. ${ }^{40}$ In its simplest form, the Förster transfer rate (in $\mathrm{ps}^{-1}$ ) is given by ${ }^{36,10}$

$$
k=1.18 J^{2} \Theta
$$

Here, $J$ is the donor-acceptor electronic coupling (in $\mathrm{cm}^{-1}$ ), and $\Theta$ is the spectral overlap integral between donor fluorescence and acceptor absorption line shapes, each normalized to a unit area on the $\mathrm{cm}^{-1}$ scale. More accurate calculations must explicitly include the vibrational manifolds and Franck-Condon factors as well as perform a proper averaging of the overlap over inhomogeneous broadening. ${ }^{45,87,88}$ (An average of the product of both line shapes rather than the product of averages is required.) In the following discussion, we use the simplest form given by eq 6.1 to gain a qualitative insight into the rates.

Estimates of spectral overlaps in B800-B800, B800-B850, and Lyc-Bchl are available ${ }^{36,34}$ (column 2 of Table 5). By substituting the calculated electronic couplings and spectral overlaps into eq 6.1 we obtained the energy transfer rates summarized in Table 5.

We first discuss Bchl-Bchl energy transfer rates between $Q_{y}$ states. The protein environment slows the B800-B800 total transfer rate by a factor of 2.4. Because the energy transfer rate quadratic in the transition dipoles $\left(k \approx J^{2} \approx d^{4}\right)$ and solvent effects reduce the $Q_{y}$ transition dipole magnitude by a factor of $\sim 1.2$, we can immediately rationalize the dielectric medium effect on B800-B800 energy transfer. Our calculated B800B850 time scales also show a similar trend with a smaller $(\sim 1.6)$ decrease due to solvent. All calculated total transfer rates are in good agreement with experiment: our computed isolated complex and dielectric medium time scales are somewhat faster and slower than experiment, respectively. The larger B800B850 electronic couplings predicted by the CEO (see Table 2) reproduce the experimental Förster hopping rates without invoking any additional mechanisms (e.g., upper exciton band 
TABLE 5. Major Energy Transfer Rates (eq 6.1) between B800-B800, B800-B850, Lyc-B850, and Lyc-B800 Chromophores in LH2 Complex of Rs. Molischianum Calculated with Couplings Obtained in the Dielectric Medium (for the Isolated Complex $)^{h}$

\begin{tabular}{|c|c|c|c|}
\hline $\begin{array}{l}\text { donor/acceptor } \\
\text { transition }\end{array}$ & $\begin{array}{l}\text { overlap } \Theta \\
\quad(\mathrm{cm})\end{array}$ & $\begin{array}{l}\text { calculated rate }{ }^{-1} \\
(\mathrm{ps})\end{array}$ & $\begin{array}{c}\text { experimental } \text { rate }^{-1} \\
(\mathrm{ps})\end{array}$ \\
\hline B800-B800 & \multirow[t]{3}{*}{$0.0032^{a}$} & $0.49(0.21)$ & \multirow[t]{3}{*}{$0.8-0.9^{d}\left(0.3-0.5^{e}\right)$} \\
\hline $1-2$ & & $1.03(0.42)$ & \\
\hline $1-3$ & & 20.4 (21.6) & \\
\hline B800-B850 & \multirow[t]{6}{*}{$0.0004^{a}$} & $0.70(0.44)$ & \multirow[t]{6}{*}{$0.6-0.7^{f}$} \\
\hline $1-1 \alpha$ & & $31.5(10.8)$ & \\
\hline $1-1 \beta$ & & $3.38(1.32)$ & \\
\hline $1-2 \alpha$ & & $1.62(0.78)$ & \\
\hline $1-2 \beta$ & & $18.9(5.5)$ & \\
\hline Lyc-B850 & & & \\
\hline $1 B_{u}-Q_{x}$ & \multirow[t]{5}{*}{$0.00021^{b}$} & $0.21(0.37)$ & \\
\hline $2 \mathrm{~L}-3 \alpha$ & & $0.31(0.68)$ & \\
\hline $2 \mathrm{~L}-1 \beta$ & & 20.6 (23.9) & \\
\hline $2 \mathrm{~L}-2 \beta$ & & $1.75(2.65)$ & \\
\hline $2 \mathrm{~L}-3 \beta$ & & $1.33(1.29)$ & \\
\hline $1 B_{u}-Q_{y}$ & \multirow[t]{5}{*}{$0.000016^{b}$} & $1.45(1.11)$ & \\
\hline $2 \mathrm{~L}-2 \alpha$ & & $21.2(17.5)$ & \\
\hline $2 \mathrm{~L}-3 \alpha$ & & $5.75(4.80)$ & \\
\hline $2 \mathrm{~L}-2 \alpha$ & & $23.0(18.9)$ & \\
\hline $2 \mathrm{~L}-3 \beta$ & & $2.78(2.12)$ & \\
\hline $2 A_{g}-Q_{y}$ & \multirow[t]{4}{*}{$0.000027^{c}$} & $1.72(1.55)$ & \\
\hline $2 \mathrm{~L}-2 \alpha$ & & $34.1(32.1)$ & \\
\hline $2 \mathrm{~L}-3 \beta$ & & $1.87(1.96)$ & \\
\hline \multicolumn{3}{|l|}{ Lyc-B800 } & \\
\hline $1 B_{u}-Q_{x}$ & \multirow[t]{3}{*}{$0.00021^{b}$} & $0.34(1.04)$ & \\
\hline $2 \mathrm{~L}-1$ & & $0.37(1.75)$ & \\
\hline $2 \mathrm{~L}-2$ & & $7.63(3.11)$ & \\
\hline $1 B_{u}-Q_{y}$ & \multirow[t]{3}{*}{$0.000016^{b}$} & $4.86(5.74)$ & \\
\hline $2 \mathrm{~L}-1$ & & $8.71(13.3)$ & \\
\hline $2 \mathrm{~L}-2$ & & $12.5(11.8)$ & \\
\hline $2 A_{g}-Q_{y}$ & \multirow[t]{6}{*}{$0.000027^{c}$} & $1.99(1.64)$ & \\
\hline $2 \mathrm{~L}-1$ & & $2.06(1.70)$ & \\
\hline total Lyc-Bchl & & & \\
\hline $1 B_{u}-Q_{y}, Q_{x}$ & & $0.12(0.21)$ & \\
\hline $2 A_{g}-Q_{y}$ & & $0.92(0.80)$ & \\
\hline depopulation of & & $0.063(0.082)$ & $0.05-0.15^{g}$ \\
\hline
\end{tabular}

$$
1 B_{u} \text { in Lyc }
$$

${ }^{a}$ Ref 36, Spectral overlap estimated for Rps. acidophila. ${ }^{b}$ Ref 34, Spectral overlap estimated for Rps. acidophila. ${ }^{c}$ Ref 49, Spectral overlap estimated for Rs. molischianum. ${ }^{d}$ Ref 10, Absorption anisotropy decay in Rs. molischianum. ${ }^{e}$ Ref 10,21,30; Femtosecond studies of Rps. acidophila. ${ }^{f}$ Ref 19-21, Femtosecond studies of Rs. sphaeroides and Rps. acidophila. ${ }^{g}$ Ref 75,35,91; Femtosecond studies of Rs. sphaeroides and Rps. acidophila. ${ }^{h}$ The chromophore labeling and electronic coupling are shown in Figures 3, 4, and 5 and Tables 2, 3, and 4 . The total transfer rates are obtained by summing the rates over all possible channels. Available experimental transfer rates are given as well. The total $1 B_{u}$ exciton depopulation rate in Lyc is calculated using $1 B_{u}-2 A_{g}$ internal conversion time of $135 \mathrm{fs}(35)$.

transfer ${ }^{30,36,10}$ and super-exchange type coupling through carotenoids ${ }^{34}$ ) which have been suggested to explain this faster transfer. Our computed total B800-B850 energy transfer rate using rates between individual chromophores may be further enhanced by Frenkel exciton delocalization in the B850 ring (see discussion below).

We next consider the energy transfer pathways from the carotenoids to Bchls- $a$, which have been the subject of extensive debates. ${ }^{10,34,49}$ Spectral overlap of $1 B_{u}-Q_{x}$ is much larger than that of $1 B_{u}-Q_{y}$ (Table 5), whereas the corresponding electronic couplings have about the same value (Table 2). This obviously makes the $1 B_{u}-Q_{x}$ channel preferable in intermolecular energy transfer: 0.13 ps $(0.27 \mathrm{ps}$ for an isolated complex $)$. In our calculations, this pathway is $\sim 9(\sim 3.5$ for an isolated complex $)$ times faster than the $1 B_{u}-Q_{y}$ channel: $1.12 \mathrm{ps}(0.93 \mathrm{ps}$ for an isolated complex) and finally accounts for $90 \%$ (80\% for an isolated complex) of total Lyc-Bchl transfer. Even though the Lyc-Bchl- $a$ spectral overlaps are smaller than those of Bchl$\mathrm{Bchl}$, the resulting energy transfer rates are faster. The computed total Lyc-B850 and Lyc-B800 time scales of 0.18 ps (0.27 ps for an isolated complex $)$ and $0.32 \mathrm{ps}(0.88 \mathrm{ps}$ for an isolated complex), respectively, are very short. Comparison of these transfer rates in two intermolecular channels (Lyc $1 B_{u}-\mathrm{B} 850$ and Lyc $\left.1 B_{u}-\mathrm{B} 800-\mathrm{B} 850\right)$ gives $65 \% / 35 \%$ (77\%/23\% for an isolated complex) branching ratio of energy migration through these pathways. We cannot directly compare this value with the total $75 \% / 25 \%$ ratio observed in several experiments ${ }^{37,38}$ because the internal conversion channel (Lyc $1 B_{u}-$ Lyc $2 A_{g}$ - Bchl) and efficiency of Lyc $1 B_{u}-$ B800 - B850 pathway are left unaccounted for (taking into account, for example, an overall efficiency of $75 \%$ for Lyc $1 B_{u}-$ B800 - B850 energy transfer suggested in (19) and unit efficiency of Lyc $1 B_{u}-$ B850 suggested by steady-state measurements in (37), we obtain $71 \%$ / $29 \%(81 \% / 19 \%$ for an isolated complex) ratio). However, the predicted trends are consistent with experiment. The total Lyc $1 B_{u}-$ Bchl- $a$ intermolecular transfer rate of $0.12 \mathrm{ps}(0.21 \mathrm{ps}$ for an isolated complex) is comparable to Lyc $1 B_{u}-2 A_{g}$ internal conversion rate $(0.135 \mathrm{ps}) .{ }^{35}$ Comparison of these time scales gives 53\%/47\% (40\%/60\% for an isolated complex) branching ratio of energy transfer through these pathways. The total $1 B_{u}$ state depopulation rate of $0.063 \mathrm{ps}$ agrees well with experiment. We next discuss the dielectric medium impact on Lyc-Bchl energy transfer rates. The total solvent effect is opposite to that in the Bchl-Bchl pathways, i.e., the transfer rate increases by a factor of 1.75 (time scale changing from 0.21 to $0.12 \mathrm{ps}$ ). However, the inaccuracy of the isolated complex computations (very large rescaling factors for Lyc $1 B_{u}-Q_{x}$ couplings are needed to match experiment, see the discussion in Section V) does not make this trend uniform for all couplings between an arbitrary pairs of chromophores (compare the respective dielectric medium and isolated complex results in Table 5).

The internal conversion channel (Lyc $1 B_{u}-$ Lyc $2 A_{g}-$ Bchl) undoubtedly plays an important role in Lyc-Bchl energy transfer. Our calculated Lyc $2 A_{g}-$ B850 and Lyc $2 A_{g}-$ B800 rates enhanced by strong spectral overlap are very fast. The total Lyc $2 A_{g}-\mathrm{B} 800$ rate of $0.92 \mathrm{ps}(0.8 \mathrm{ps}$ for an isolated complex $)$ is much faster than the experimentally measured $9.1 \mathrm{ps}$ lifetime of carotenoid ${ }^{19}$ and leads to the very highly efficient energy flow process. We note that, overall, the solvent slightly decreases the transfer time scales. Comparison of Lyc $2 A_{g}-\mathrm{B} 850$ and Lyc $2 A_{g}-$ B800 time scales gives 54\%/46\% (52\%/48\% for an isolated complex) ratio of relative distribution of energy going through these pathways, i.e., the flow splits almost equally. However, smaller total efficiency of Lyc $2 A_{g}-$ B800 - B850 compared to the direct channel and increase of $2 A_{g}-\mathrm{B} 850$ process efficiency due to delocalization of Frenkel exciton in the B850 ring (see discussion below) may favor the direct channel.

Finally, we discuss a possible enhancement of energy transfer to the $Q_{y}$ band in the B850 ring due to exciton delocalization. Suppose an excitation is transferred from Lyc ring or B800 ring to $\mathrm{B} 850$ pigments and $J_{j}, j=1,16$ are the corresponding couplings to each B850 chromophores. If an excitation is transferred between individual molecules then the total transfer rate (eq 6.1) is

$$
k_{i}=1.18 \Theta \sum_{j}^{16} J_{j}^{2}
$$

On the other hand, if the $Q_{y}$ band of B850 is made out of delocalized Frenkel excitons, the degree of exciton delocalization 
is determined by the competition between intermolecular interactions and energetic disorder originating from slow protein motions. Assuming that the energy transfer is slow compared to the energy relaxation on all components and the baths are uncorrelated, the energy transfer rate $k_{e}$ from a single chromophore of $\mathrm{B} 800$ or $\mathrm{Lyc}$ ring to the $\mathrm{B} 850$ ring can be represented in a form

$$
k_{e}=\sum_{i j} J_{i} J_{j} \int_{0}^{\infty} \frac{d \omega}{2 \pi} \operatorname{Im}\left[G_{i j}(\omega)\right] f(\omega)
$$

In eq $6.3, f(w)$ is the fluorescence spectrum of a single chromophore in B800 or Lyc ring normalized to a unit area

$$
\int_{0}^{\infty} \frac{d \omega}{2 \pi} f(\omega)=1
$$

whereas $G_{i j}(\omega)$ is the exciton Green function of the B850 ring in the frequency domain

$$
G_{i j}(\omega) \equiv \int_{0}^{\infty} d t e^{i \omega t}\left\langle B_{i}(t) B_{j}^{+}(0)\right\rangle
$$

Assuming that the disorder is stronger than the vibronic coupling, which is typically the case in antennae complexes, and applying the theory developed in (89) for the exciton Green function, eq 6.3 adopts the form

$$
k_{e}=\sum_{m n \alpha} J_{m} J_{n} \varphi_{\alpha}(m) \varphi_{\alpha}(n) \int_{0}^{\infty} \frac{d \omega}{2 \pi} g_{\alpha}(\omega) f(\omega)
$$

Here, $\varphi_{\alpha}(m)$ are the exciton wave function, and $g_{\alpha}(\omega)$ are the single exciton absorption spectra normalized to a unit area. Equation 6.6 explicitly takes into account the diagonal vibronic coupling in the exciton basis set and neglects the nuclear motions induced coupling between different excitons. It has been demonstrated ${ }^{89}$ that this approximation holds provided disorderinduced localization effects are strong compared to polaron formation.

Equation 6.6 shows that the energy transfer rate results from an interplay of the exciton shapes $\varphi_{\alpha}(m)$, the couplings $J_{m} J_{n}$, the single-exciton absorption spectra $g_{\alpha}(\omega)$ and the singlechromophore fluorescence spectrum $f(\omega)$. The overlap of $f(\omega)$ and $g_{\alpha}(\omega)$ in eq 6.6 limits the excitons participating in the energy transfer to a certain spectral region. On the other hand, the exciton shape $\varphi_{\alpha}(m)$ strongly depends on its energy: low energy (band edge) exciton wave functions are nonoscillatory and strongly localized, in contrast to the less localized, highly oscillatory, exciton wave functions inside the exciton band. It has been demonstrated ${ }^{41,42}$ that even though the participation ratio shows a localization length of $4-5$ chromophores, for the lower-energy excitons, the number of chromophores coupled coherently is about the aggregate size. On the other hand, it follows from the calculations presented in this paper that the coupling factors $J_{i} J_{j}$ are localized on 4 chromophores. The coupling pattern calculated in this paper constitutes a key ingredient for a detailed analysis of the energy transfer rates distribution based on eq 6.6. More accurate computations are needed to address the issue of exciton delocalization. ${ }^{41,43,10}$

In summary, we have reported the effective Frenkel exciton Hamiltonian in the LH2 complex of Rs. molischianum (Tables 2, 3, 4 and Figures 3, 4, 5). Electronic couplings among all 24 pigments (B800, B850, and carotenoid: 8, 16, and 8, respectively) are computed for an isolated complex and in a dielectric medium using two different approaches that allow us to separate the Coulombic (Förster) and electron exchange (Dexter) con- tributions to interchromophore interactions. The latter contribution accounts for approximately $10-15 \%$ of the interaction between nearest neighbor Bchls- $a$ in the upper B850 ring and is negligible among all other pigments. The protein environment affects significantly the Bchl- $a$ absorption spectrum and therefore also the intermolecular interactions. Using the calculated couplings, we have estimated B800-B800, B800-B850, LycB800, and Lyc-B850 Förster energy transfer rates (Table 5). The Bchl-Bchl time scales agree well with experiment, and the intermolecular Lyc-Bchl transfer rate from $1 B_{u}$ is found to be comparable to the $1 B_{u}-2 A_{g}$ internal conversion rate. Overall, the dielectric environment slows down the Bchl-Bchl and Lyc $2 A_{g}-\mathrm{Bchl}$, and increases the Lyc $1 B_{u}-\mathrm{Bchl}$ energy transfer channels.

Acknowledgment. This article is dedicated to the memory of Prof. M. Zerner, whose seminal contributions have always been an invaluable source of inspiration. We wish to thank Prof. K. Schulten and Prof. M. Zerner for providing us with crystal structures of Rs. molischianum. S.T. gratefully acknowledges the support of a LANL Director's Postdoctoral Fellowship and the LDRD program at LANL. The support of the National Science Foundation and the Petroleum Research Fund, sponsored by the American Chemical Society, is gratefully acknowledged.

\section{References and Notes}

(1) Zuber, H.; Brunisholz, R. A.; Photosynthesis: Physical Mechanism and Chemical Patterns; Cambridge University Press: New York, 1980.

(2) Zuber, H.; Brunisholz, R. A. In Chlorophylls; Scheer, H., Ed.; CRC, Boca Raton, FL, 1991; 1047.

(3) McDermott, G.; Prince, S. M.; Freer, A. A.; HawthornthwaiteLawless, A. M.; Papiz, M. Z.; Cogdell, R. J.; W Isaacs, N. Nature 1995, 374, 517.

(4) Koepke, J.; Hu, X.; Muenke, C.; Schulten, K.; Michel, H. Structure 1996, 4, 581.

(5) Karrasch, S.; Bullough, P. A.; Gosh, R. EMBO J. 1995, 14, 631. 614.

(6) Kuhlbrandt, W.; Wang, D. N.; Fujiyoshi, Y. Nature 1994, 367,

(7) Savage, H.; Cyrklaff, M.; Montoya, G.; Kuhlbrandt, W.; Sinning, I.; Structure 1996, 4, 243.

(8) Miller, K. Nature 1982, 300, 53

(9) Pullerits, T.; Sundström, V. Acc. Chem. Res. 1996, 29, 381.

(10) Pullerits, T.; Sundström, V.; van Grondelle, R. J. Phys. Chem. B. 1999, 103, 2327.

(11) Fleming, G. R.; van Grondelle, R. Phys. Today 1994, 47, 48.

(12) Hu, X. C.; Damjanovic, A.; Ritz, T.; Schulten, K. Proc. Natl. Acad. Sci. U.S.A. 1998, 95, 5935 .

(13) van Grondelle, R.; Dekker, J. P.; Gillbro, T.; Sundström, V. Biochim. Biophys. Acta 1994, 1187, 1.

(14) Sundström, V.; van Grondelle, R. In Anoxigenic Photosynthetic Bacteria; Blankenship, R. E., Madiga, M. T., Baner, C. E., Eds.; Kluwer Academic: Drodrecht, 1995; p 349.

(15) Hu, X. C.; Schulten, K. Phys. Today 1997, 50, 28.

(16) Sauer, K.; Cogdell, R. J.; Prince, S. M.; Freer, A. A.; Isaacs, N. W.; Scheer, H. Photochem. Photobiol. 1996, 64, 564.

(17) Alden, R. G.; Johnson, E.; Nagarajan, V.; Parson, W. W.; Law, C. J.; Cogdell, R. G. J. Phys. Chem. B 1997, 101, 4667.

(18) Monshouwer, R.; Abrahamsson, M.; van Mourik, F.; van Grondelle, R. Phys. Chem. B 1997, 101, 7241.

(19) Shreve, A. P.; Trautman, J. K.; Frank, H. A.; Owens, T. G.; Albrecht, A. C. Biochim. Biophys. Acta 1991, 280, 1058.

(20) Ma, Y.-Z.; Cogdell, R. J.; Gillbro, T. J. Phys. Chem. B 1997, 101, 1087.

(21) Ma, Y.-Z.; Cogdell, R. J.; Gillbro, T. J. Phys. Chem. B 1998, 102, 881 .

(22) Joo, T.; Jia, Y.; Yu, J.-Y.; Jonas, D. M.; Fleming, G. R. J. Phys. Chem. 1996, 100, 2399.

(23) Reddy, N. R. S.; Picorel, R.; Small, G. J. J. Phys. Chem. 1992, 96, 9458.

(24) Pullerits, T.; Chachisvilis, M.; Sundström, V. J. Phys. Chem. 1996, 100,10787

(25) Hess, S.; Feldchtein, F.; Babin, A.; Nurgaleev, I.; Pullerits, T.; Sergeev, A.; Sundström, V. Chem. Phys. Lett. 1993, 216, 247. 
(26) Monshouwer, R.; de Zarate, I. O.; van Mourik, F.; van Grondelle, R. Chem. Phys. Lett 1995, 246, 341.

(27) Chachisvilis, M.; Fidder, H.; Pullerits, T.; Sundström, V. J. Raman Spectrosc. 1995, 26, 513 .

(28) Chachisvilis, M.; Kühn, O.; Pullerits, T.; Sundström, V. J. Phys. Chem. B 1997, 101, 7275 .

(29) Nagarajan, V.; Alden, R. G.; Williams, J. C.; Parson, W. W.; Proc. Natl. Acad. Sci. U.S.A. 1996, 93, 13774.

(30) Wu, H.-M.; Savikhin, S.; Reddy, N. R. S.; Jankowiak, R.; Cogdell,

R. J.; Struve, W. S.; Small, G. J. J. Phys. Chem. 1996, 100, 12022.

(31) Kumble, R.; Hochstrasser, R. J. Chem. Phys. 1998, 109, 855.

(32) Jimenez, R.; van Mourik, F.; Fleming, G. R. J. Phys. Chem. B 1997, 101,7350 .

(33) Wasielewski, M. R.; Tiede, D. M.; Frank, H. A. In Ultrafast Phenomena; Fleming, G. R., Siegman, A. E., Eds.; Springer-Verlag: Berlin, 1986; pp 388-392.

(34) Krueger, B. P.; Scholes, G. D.; Fleming, G. R. J. Phys. Chem. B 1998, 102,5378 .

(35) Krueger, B. P.; Scholes, G. D.; Jiminez, R.; Fleming, G. R. J. Phys. Chem. B 1998, 102, 2284.

(36) Pullerits, T.; Hess, S.; Herek, J. L.; Sundström, V. J. Phys. Chem. B 1997, 101, 10560 .

(37) van Grondelle, R.; Kramer, H. J. M.; Rijgersberg, C. P. Biochim. Biophys. Acta 1982, 682, 208. Kramer, H. J. M.; van Grondelle, R.; Hunter, C. N.; Westerhuis, W. H. J.; Amesz, J. Biochim. Biophys. Acta 1984, 765, 156.

(38) Chadwick, B. W.; Zhang, C.; Cogdell, R. J.; Frank, H. A.; Biochim. Biophys. Acta 1987, 893, 444.

(39) Scholes, G. D.; Gould, I. R.; Cogdell, R. J.; Fleming, G. R. J. Phys. Chem. B 1999, 103, 2543.

(40) Förster, Th. Naturwissenschaften 1946, 33, 166 7332 .

(41) Meier, T.; Chernyak, V.; Mukamel, S. J. Phys. Chem. B 1997, 101,

(42) Meier, T.; Zhao, Y.; Chernyak, V.; Mukamel, S. J. Chem. Phys. 1997, 107, 3876.

(43) Meier, T.; Chernyak, V.; Mukamel, S. J. Chem. Phys. 1997, 107, 8759.

(44) Pearlstein, R. M. In Chlorophylls; Scheer, H., Ed.; CRC: Boca

Raton, FL, 1991; 1047.

(45) Scholes, G. D.; Fleming, G. R. J. Phys. Chem. B 104, 18542000.

(46) Linnanto, J.; Korppi-Tommola, J. E. I.; Helenius, V. M. J. Phys.

Chem B. 1999, 103, 8739

(47) Hu, X.; Ritz, T.; Damjanovic, A.; Schulten, K. J. Phys. Chem.,

1997, 101, 3854.

(48) Cory, M. G.; Zerner, M. C.; Hu, X.; Schulten, X. K.; J. Phys. Chem., 1998, $102,7640$.

(49) Damjanovic, A.; Ritz, T.; Schulten, K. Phys. Rev. E 1999, 59, 3293.

(50) Tretiak, S.; Middleton, C.; Chernyak, V.; Mukamel, S. J. Phys. Chem., 104, 4519, 2000.

(51) Tretiak, S.; Chernyak, V.; Mukamel, S. Chem. Phys. Lett. 1996 259, 55. Tretiak, S.; Chernyak, V.; Mukamel, S. J. Chem. Phys. 1996, 105, 8914.

(52) Tretiak, S.; Chernyak, V.; Mukamel, S. J. Am. Chem. Soc. 1997, 119, 11408

(53) Mukamel, S.; Tretiak, S.; Wagersreiter, T.; Chernyak, V. Science 1997, 277, 781.

(54) Tretiak, S.; Chernyak, V.; Mukamel, S. J. Phys. Chem. B 1998 102,3310 .

(55) Tretiak, S.; Zhang, W. M.; Chernyak, V.; Mukamel, S. Proc. Nat. Acad. Sci., 1999, 96, 13003.

(56) Bazan, G. C.; Oldham, W. J. Jr.; Lachicotte, R. J.; Tretiak, S.; Chernyak, V.; Mukamel, S. J. Am. Chem. Soc., 1998, 120, 9188.

(57) Tretiak, S; Chernyak, V.; Mukamel, S. Chem. Phys. Lett. 1998, 297, 357.

(58) Pople, J. A.; Segal, G. A. J. Chem. Phys., 43, S136, 1965.

(59) Pople, J. A.; Beveridge, D. L.; Dobosh, P. J. Chem. Phys. 1967 47, 2026.

(60) Ridley, J.; Zerner, M. C. Theor. Chim. Acta 1973, 32, 111.

(61) Zerner, M. C.; Loew, G. H.; Kirchner, R. F.; Mueller-Westerhoff, U. T. J. Am. Chem. Soc. 1980, 102, 589.
(62) Dewar, M. J. S.; Zoebisch, E. G.; Healy, E. F.; Stewart, J. J. P. J Am. Chem. Soc. 1985, 107, 3902.

(63) Szabo, A.; Ostlund, N. S. Modern Quantum Chemistry: Introduction to Advanced Electronic Structure Theory; McGraw-Hill: New York, 1989.

(64) McWeeny, R.; Sutcliffe, B. T. Methods of Molecular Quantum Mechanics; Academic Press: New York, 1976.

(65) Davidson, E. R. Reduced Density Matrixes in Quantum Chemistry; Academic Press: New York, 1976.

(66) In earlier studies we have used the Density Matrix Spectral Moment Algorithm for computing the eigenmodes (51). That algorithm is particularly suitable for off resonant static response computations where only transitions with nonzero oscillator strengths are calculated. We found that the oblique Lanczos algorithm is more efficient and provides a more accurate representation of the resonant response than the DSMA.

(67) Chernyak, V; Tretiak, S.; Schulz, M.; Tsiper, E. V.; Mukamel, S. J. Chem. Phys. 2000, 113, 36.

(68) Karlsson, G.; Zerner, M. C. Int. J. Quantum Chem. 1973, 7, 35.

(69) Karlsson, G.; Zerner, M. C. J. Phys. Chem. 1992, 96, 6949.

(70) King, G.; Lee, F.; Warshel, A. J. J. Chem. Phys. 1991, 95, 4366.

(71) Kleima, F. J.; Wendling, M.; Hofmann, E.; Peterman, E. J. G.; van Grondelle, R.; van Amerongen, H. Biochemistry 2000, 39, 5184.

(72) Frisch, M. J.; et al. Gaussian 98 (Revision A.7); Gaussian, Inc.: Pittsburgh, PA, 1998.

(73) Oelze, J. Methods Microbiol. 1985, 18, 257.

(74) Sauer, K. Lindsay Smith, J. R.; Shultz, A. J. J. Am. Chem. Soc. 1966, $88,2681$.

(75) Andersson, P. O.; Gillbro, T.; Ferguson, L.; Cogdell, R. J. Photochem. Photobiol. 1991, 54, 353.

(76) Baker, J. D.; Zerner, M. C. Chem. Phys. Lett. 1990, 175, 192

(77) Soos, Z. G.; Galvao, D. S.; Etemad, S. Phys. Rev. B 1993, 47, 1742. Soos, Z. G.; Ramasesha, S.; Galvao, D.; Kepler, R.; Etemad, S. Synth Metals 1993, 54, 35. Mukhopadhyay, D.; Hayden, G. W.; Soos, Z. G. Phys. Rev. $B$ 1995, 51, 15.

(78) Koyama, Y.; Kuki, M.; Andersson, P.; Gillbro, T. Photochem Photobiol. 1996, 63, 243.

(79) Davydov, A. S. Theory of Molecular Excitons; Plenum: New York, 1971. Pope, M.; Swenberg, C. E. Electronic Processes in Organic Crystals, 2nd ed; Oxford University Press: Oxford, New York, 1999.

(80) Kasha, M.; Rawls, H. R.; Ashraf El-Bayoumi, M. Pure Appl. Chem. 1965, 11, 371 .

(81) Rashba, E. I., Sturge, M. D., Eds. Excitons; North-Holland: Amsterdam, 1982. Broude, V. B.; Rashba, E. I.; Sheka, E. F. Spectroscopy of Molecular Excitons; Springer: Berlin, 1985.

(82) Poliakov, E.; Chernyak, V.; Tretiak, S.; Mukamel, S. J. Chem. Phys. 1999, 110, 8161. Minami, T.; Tretiak, S.; Chernyak, V.; Mukamel, S. J. Lumin. 2000, 87-9, 115.

(83) Siddiqui, S.; Spano, F. C. Chem. Phys. Lett. 1999, 308, 99

(84) Kumble, R.; Palese, S.; Visschers, R. W.; Dutton, P. L.; Hochstrasser, R. M. Chem. Phys. Lett. 1996, 261, 396.

(85) Arnett, D. C.; Moser, C. C.; Dutton, P. L.; Scherer, N. F. J. Phys. Chem. B 1999, 103, 2014.

(86) Novoderezhkin, V.; Monshouwer, R.; van Grondelle, R. J. Phys. Chem. B 1999, 103, 10540.

(87) Wu, H.-M.; Savikhin, S.; Reddy, N. R. S.; Jankowiak, R.; Cogdell, R. J.; Struve, W. S.; Small, G. J. J. Phys. Chem. 1996, 100, 12022.

(88) Small, G. J.; Hayes, J. M.; Silbey, R. J. J. Phys. Chem. 1992, 96, 7499. Kolaczkowski, S. V.; Hayes, J. M.; Small, G. J. J. Phys. Chem. 1994, 98, 13418.

(89) For a general discussion of this representation, see eq 4.36 in Mukamel, S. Principles of Nonlinear Optical Spectroscopy; Oxford, New York, 1995.

(90) Koolhaas, M. H. C.; Frese, R. N.; Fowler, G. J. S.; Bibby, T. S.; Georgakopoulou, S.; van der Zwan, G.; Hunter, C. N.; van Grondelle, R.; Biochemistry 1998, 14, 4693.

(91) Krueger, B. P.; Yom, J.; Walla, P. J.; Fleming, G. R.; Chem. Phys. Lett., 1999, 310, 57, and references therein. 